\title{
DISCLAIMER
}

This report was prepared as an account of work sponsored by an agency of the United States Government. Neither the United States Government nor any agency thereof, nor any of their employees, makes any warranty, express or implied, or assumes any legal liability or responsibility for the accuracy, completeness, or usefulness of any information, apparatus, product, or process disclosed, or represents that its use would not infringe privately owned rights. Reference herein to any specific commercial product, process, or service by trade name, trademark, manufacturer, or otherwise does not necessarily constitute or imply its endorsement, recommendation, or favoring by the United States Government or any agency thereof. The views and opinions of authors expressed herein do not necessarily state or reflect those of the United States Government or any agency thereof.

\section{MULTIPLY STRIPPED ION GENERATION IN THE METAL VAPOR VACUUM ARC*}

I. G. Brown, B. Feinberg and J. E. Galvin

\author{
Lawrence Berkeley Laboratory \\ University of California \\ Berkeley, CA 94720
}

August 1986 *This work was supported by the U.S. Department of Energy under Contract No.
$D E-A C 03-76 S F 00098$. 


\section{ABSTRACT}

We consider the charge state distribution of ions produced in the metal vapor vacuum arc plasma discharge. A high current metal ion source, the MEVVA ion source, in which the ion beam is extracted from a metal vapor vacuum arc plasma, has been used to obtain the spectra of multiply charged ions produced within the cathode spots. A computer calculation of the charge state distribution that evolves within the spots via stepwise ionization of ions by electron impact provides a theoretical basis for comparison of the data. In this paper we report on the measured charge state distributions for a wide variety of metallic species and compare these results with the predictions of this theory. 


\section{INTRODUCTION}

The metal vapor vacuum arc is a plasma discharge that occurs between conducting electrodes in vacuum. As the arc proceeds, material is evolved from the electrodes, mostly from the cathode so long as the arc current is not t.oo high, and a dense metal plasma is created. The fundamental phenomenon which drives the vacuum arc is that of cathode spot formation - minute regions of intense current concentration which reside on the surface of the cathode and at which the solid cathode material is vaporized, ionized, and injected into the interelectrode arc region. The current density at the cathode spots is of order $10^{6} \mathrm{Amps} / \mathrm{cm}^{2}$ over a spot size of order microns. A typical vacuum arc discharge might consist of from one to many dozens of such spots. In general a spot will be in vigorous motion on the cathode surface, and will have a lifetime of order microseconds. It is within the intense fireball of the cathode spot that the plasma constituents of the arc are formed - the parameters of the arc are in large part determined by the plasma physics of the spots. Thus an understanding of the cathode spot plasma is essential to any attempt to use or control the arc as a plasma device.

The study of the metal vapor vacuum arc discharge - also called the vacuum arc or metal vapor arc - had its origin in the high power switching field, where it found application as a high voltage switch in a vacuum environment. One of the earliest publications in the field is that of Sorensen and Mendenhall in 1926 [1]; early work was severely impeded by the rudimentary vacuum lechniques of the era. An historical survey of the field, pre-1960s, has been given by Cobine [2]. More recently a very complete review of the entire field of metal yapor arc discharges has been given by Lafferty [3], and a review of cathode spot behavior has been given by Lyubimov and Rakhovskii [4].

The production of ions in the metal vapor vacuum arc plasma has been investigated by a number of authors over at least the last two decades [5-18]. One of the earliest attempts to incorporate this $k$ ind of arc as the plasma formation mechanism for use as an ion source was the work done as part of the Manhattan Project in World War 11 [19]; the source suffered from several drawbacks and this work was abandoned. Revutskij et al [20], in 1968, described a cylindrically symmetric arc geometry employing ion extraction 
through a hole in the cathode (as opposed to through the anode, as in our work, to be described), and their work appears not to have been pursued. More recently, sources of this kind have been described by Adler and Picraux [21], and by Humphries and coworkers [22-24].

We have developed an ion source in which the metal vapor vacuum arc is used as the method of plasma production and from which high quality, high current beams of metal ions can be extracted [25-28]. We have called this source the MEVVA ion source, as an acronym for the mechanism employed. The source is described below. With this source we have produced beams at voltages up to $100 \mathrm{kV}$ and with ion current up to 1 Ampere. The source works well with a wide range of ion species, spanning the periodic table from lithium to uranium. In general, for elements not too low on the periodic table, the ions produced are multiply ionized.

The average charge state is higher for higher $z$ elements, and to a lesser extent for higher arc current. For example, a uranium beam typically is composed of species with charge state from $Q=2$ to 6 , a chromium beam has charge states $Q=1,2$ and 3 , and a lithium beam consists of the singly ionized $Q=1$ species only. For almost all applications of the source, there is considerable advantage to a beam with ions stripped maximally. Hence our interest in understanding the physics of the MEVVA charge slate distribution and in trying to achieve upwards control over the distribution.

Measurements of the charge state distribution of ions generaled by the vacuum arc have been reported by a number of workers [5,6,29-32], and it is well recognized that the distributions in general contain a high fraction of multiply stripped species. Theoretical understanding of the cathode spot plasma is, however, very incomplete. 
The MEVVA ion source has been described elsewhere [25-28]. Briefly, in this source we make use of the intense plume of highly ionized metal plasma that is created at the cathode spots of a metal vapor vacuum arc discharge to provide the "plasma feedstock" from which the ion beam is extracted. The quasi-neutral plasma plumes away from the cathode toward the anode and persists for the duration of the arc current drive. The anode of the discharge is located on axis with respect to the cylindrical cathode and has a central hole through which a part of the plasma plume streams; it is this component of the plasma that forms the medium from which the ions are extracted. The plasma plume drifts through the post-anode region to the set of grids that comprise the extractor - a three grid, accel-decel, multi-aperture design. A small axial magnetic field of up to about 100 gauss produced by a simple coil surrounding the arc region serves to help duct the plasma plume in the forward direction, but this is not essential to the source operation.

A schematic of the embodiment of the concept with which we've done most of our work is shown in figure 1 . This is the device called MEVVA LI. The various components and features referred to above can be seen. The extractor diameter is $2 \mathrm{~cm}$, as is the initial beam diameter.

The arc is driven by a simple pulse line. The line is a 6-section LC network of impedance 0.5 ohms and pulse length 250 microseconds, with a modified Gibbs section on the front end to provide a fast rise to the pulse. The line is charged to a voltage of up to several hundred volts with a small. isolated, dc power supply. A high voltage pulse applied to a trigger electrode initiates a surface spark discharge between the trigger electrode and the cathode, which in turn causes the main anode-cathode circuit to close due to the spark plasma, and the vacuum arc proceeds. Typically the source is operated at a repetition rate of several pulses per second, up to a maximum of near 100 pulses per second for short pulse length and low average power; we are presently increasing the duty cycle at which the source will run. 
The source is operated on a test-stand equipped with various diagnostics to monitor the source performance and the parameters of the extracted beam. Base pressure is in the low $10^{-6}$ lorr range. The arc current is routinely monitored; the arc voltage (anode-cathode drop) is measured only when beam is not extracted, since during extraction the arc circuit is biased to the full extraction potential of several tens of kilovolts. In most of the work described here the small magnet coil surrounding the arc was not energized; the effect of this field is to increase the efficiency with which the arc plasma is converted to useable ion beam, but this was not a concern for the present work. Beam current is measured by a magnetically suppressed Faraday cup, and we have cross-checked these measurements with those obtained using several different designs of beam calorimeters. Beam divergence and emittance were measured with a 16-collector beam profile monitor [33,34] and with a "pepper pot" [35] device. We find that a beam current of several hundred milliamperes into a half-angle divergence of from $1^{0}$ to $3^{0}$, or an emittance of $<0.05 \pi \mathrm{cm}$ mrad (normalized), can be routinely produced.

The ion source, its operation, and the supporting facilities have been fully described in reference 27 , to which the reader is referred for more detail.

The charge state distribution (CSD) of the extracted ion beam has been measured using a time-of-flight (TOF) diagnostic. In this device a pair of deflection plates is located in the beam path and biased so as to deflect the beam aside except for a short pulse of from 0.1 to 1.0 microseconds in length; in this way a short sample of the beam is obtained. This short pulse is allowed to drift down a $1.6 \mathrm{~m}$ long chamber, during which drift lime the different charge-to-mass ( $Q / A)$ components of the beam separate out, since they have been accelerated through the same potential drop in the ion source extractor and thus have flight times proportional to $(Q / A)^{-1 / 2}$. A detector (an RCA 7265 photomultiplier with the front glass surface removed) at the end of the drift chamber measures the arrival time of the different Q/A components of the beam. The detector is not in a direct line-of -sight to the MEVVA ion source, so as to shield the detector from the intense visible light and UV generated by the vacuum arc; the direct path is blocked by metal plates, and the beam is steered onto the detector by the deflection plates. It is an assumption that the rof spectrum is a good measurement of the CSO. The ion 
current at the detector is very small and the electromagnetic environment is noisy, and a calibration of the sensitivity as a function of charge state has not yet been possible; to this extent the CSD is uncertain. The charge state distribution has been measured by a more conventional magnetic analysis for a few particular cases, and the agreement with the time-of-flight spectra is good. A schematic of the experimental configuration is shown in figure 2 . The measured flight times for the various charge states are well fitted by the calculated values, usually to better than the measurement uncertainty of about 1\%. The spectra are generally quite clean, with minimal impurity contamination.

\section{I. THEORETICAL MODEL}

We consider the plasma within the cathode spot and make the assumption that our measurements of charge state distribution of the MEVVA ion beam are a good indicator of the charge state distribution of ions within the cathode spots. All the ionization and stripping to higher charge states is assumed to occur within the spots and not in the plasma plume extending from cathode to anode; this has been confirmed experimentally by the observation that the charge state spectrum does not change when the cathode-anode separation is varied by a factor of two. We do not consider here the origin of the cathode spots nor the mechanism that maintains them, but only the plasma parameters that are implied by the measured charge state distributions. A comparison of the experimental results with the theoretical model outlined here yields information about the plasma parameters; this comparison and the implications are discussed in Section $V$.

Ions are created within the cathode spot plasma by ionization from the neutral state by electron impact. The plasma ions may be further stripped by a number of different processes, of which the most important is assumed to be stepwise ionization by successive electron impact [36]. Multiple ionization

the removal of several electrons in a single collision - has been examined experimentally and theoretically by Mueller [37,38]. While multiple ionization is likely to be significant at these electron temperatures for high $z(z \geq 50)$. it has not been included because of the lack of a good general model. In addition, ionization of excited states, which is also likely to be significant at these high densities, has been omitted in this simple model. 
The time history of the charge state distribution is determined by the electron energy, $E_{e}$, and the product $n_{e} \tau_{i}$ of electron density $n_{e}$ and ion residence time within the stripping region, $\tau_{j}$. Thus the plasma electrons must be sufficiently energetic to remove the bound electrons by collisions, and the plasma electron density and ion residence time within the plasma must be sufficiently great to allow the stripping to proceed. Calculations of the parameters necessary to achieve given charge states for a variety of elements have been carried out by a number of authors [39-41].

The computer code developed here integrates a set of coupled rate equations of the form

$$
\begin{aligned}
\frac{d n_{i}}{d t}= & n_{i-1} n_{e}\left\langle\sigma_{i-1, i v\rangle}-n_{j} n_{e}\left\langle\sigma_{i, i+1} v\right\rangle\right. \\
& -n_{j} n_{0}\left(R_{i, i,-1}+R_{i, j-2}\right) \\
& +n_{i+1} n_{0} R_{i+1, i}+n_{i+2} n_{0} R_{i+2, i}
\end{aligned}
$$

where $n_{i}$ is the density of ions of charge state $i, n_{e}$ is the electron density and $n_{0}$ is the background neutral density. $\sigma_{i, i+1}$ is the cross section for ionization from charge state $i$ to charge state $i+1$ by impact with electrons of velocity $v$, and the average <ov> is taken over the distribution of electron velocities. The distribution can be either mono-energetic or Maxwellian. The cross sections are taken as given by Lotz [42] using binding energies and ionization potentials given by Lotz [43,44] and Carlson et al $[45,46]$. The recombination rates $R_{j, j-1}, R_{j, j-2}$ are given by Mueller and Salzborn [47].

The initial conditions can be specified with either a constant neutral density or a neutral density that changes with time. The first case represents a steady input of neutrals from the cathode, while the second case allows these neutrals to decrease in number as they traverse the spot plasma and are ionized. Another option in the program allows the charge states to be averaged over a Gaussian density distribution; with this model the electron density is represented as a cylinder with a Gaussian radial distribution, and the neutral density input from the cathode is also given a Gaussian radial distribution. 
This kind of calculation has been carried out for comparison with the charge state distributions of ions produced in EBIS (Electron Beam Ion Source) devices. In the EBIS, ions are confined within the electrostatic well of an intense, energetic electron beam and they are stripped to high charge state by collisions with the beam electrons; these sources have been developed at a number of laboratories [48-54]. EBIS data provide a good reference with which to compare the predictions of a stripping theory, because of the well-defined electron energy, electron density, and ion residence time. Such a comparison has been made by Donets $[48,55]$. For the present work we have compared the predictions of the computer program developed here against the same EBIS data, as a check on the program. The comparison was good.

Charge state distributions have been calculated in this way for many of the cathode materials with which the MEVVA ion source has been run. The computer program provides a graph of the ion fraction in each charge state as a function of time, given the electron density and velocity distributions and other initial conditions. The results of a typical calculation are shown in Figure 3, where the time evolution of the charge state distribution for titanium is shown. A "time slice" of the charge state distribution can then be chosen for comparison with the experimental data. In this comparison the confinement time $\tau_{i}$ of the theoretical treatment is equivalent to the meari ion residence time within the cathode spot, a lower limit to which is the ion flight tilue across the spot dimension. 
The TOF spectra measured for a number of cathode elements spanning the periodic table are shown in Figures $4-21: \mathrm{C}, \mathrm{Mg}, \mathrm{Al}, \mathrm{Si}, \mathrm{Ti}, \mathrm{Cr}, \mathrm{Fe}, \mathrm{Co}, \mathrm{Nb}$, Mo, Sn, La, Gd, Ho, Ta, W, Pb, and U. These data were taken under different conditions of extractor voltage, so the particle velocities through the time-of-flight chamber and the oscillogram sweep speeds are different. The arc current was not the same for all cathode materials, but was generally in the range 200 - 400 Amps; the TOF spectrum, however, is not a strong function of arc current. The arc current controls the beam current more than its charge state distribution - as the arc current is increased, the number of cathode spots increases, but the plasma physics within a single spot remains much the same. It is evident from these spectra that one parameter that has a strong effect on the CSD is the atomic number of the cathode material. There is a strong tendency for the CSD to increase to higher average charge state with the $z$ of the material. There is also an indication that softer (lower melting point) materials have lower average charge states.

Figures 22 - 24 show the TOF spectra obtained for the case when the cathode material is a conducting compound rather than a metallic element. The spectra shown are for the refractory carbides TiC and SiC, and lead sulfide, PbS. These results are significant in several ways. Firstly, it is evident that beams containing non-metallic species can be produced; cathode spots form on the surface of the conducting cathode and the non metallic component of the molecule participates in the plasma as well as the metallic. Secondly, the ionization states of the elemental constituents of the "compound discharge" are different from those produced in the "elemental discharge". Thus $\mathrm{C}^{2 t}$ is evident in the SiC and TiC spectra, but we have never seen $c^{2+}$ from a pure carbon cathode - only the singly ionized $\mathrm{C}^{+}$; similarly $\mathrm{Si}^{3+}$ appears in the sic spectrum but we see only small amounts of $\mathrm{si}^{3+}$ from a pure silicon cathode. This effect is presumably a manifestation of the different plasma parameters of the cathode spots formed on the elemental and the compound surfaces.

Table 1 summarises all the ionization state measurements we've made to-date. In this tabulation, the percentages of the different charge states should be taken only as approximate; the spectrum varies a little with arc current, and the cases we have taken are typical for an arc current of several hundred Amps. 
It is interesting to try to predict the charge state distributions. There are two senses in which this can be done - prediction of the charge state distribution for a given cathode material, and prediction of the variation of charge state distributions for different cathode materials. These two concerns are addressed in the following.

The computer program described in Section 111 has been used to fit the measured CSDS. Binding energies are input as parameters for calculation of the Lotz cross-sections for the particular element linder consideration. The electron energy distribution can be taken either as monoenergetic, in which case the electron energy $E_{e}$ is specified as an input parameter, or as a Maxwellian, in which case the electron temperature $T_{e}$ is specified. New neutral particles can be introduced continuously, in which case all the ionization states which appear approach a non-zero asymptotic value, or the initial particle population can be allowed to evolve without input of fresh neutrals, in which case the lower charge states "burn out" as they are stripped to higher $\bar{Q}$ values and not replaced. Finally, charge exchange with background neutrals can be included or omitted.

Some examples of how the calculations fit the measured spectra are shown. Figure 25 shows the measured and calculated CSOs for titanium. The experimental data have been taken directly from figure 8 , and the calculated values from Figure 3. Parameters for the calculation were: Maxwellian electron energy dis tribution with $T_{e}=20 \mathrm{eV}$, no steady injection of fresh neutrals, no charge exchange, and $j_{e^{\top}}=2.0 \times 10^{16}$ electrons $/ \mathrm{cm}^{2}$. If we arbitrarily take the current density at the spot to be $1 \mathrm{MA} / \mathrm{cm}^{2}$ then $\tau_{i}=3.2 \mathrm{nsec}$, in which time a titanium ion of energy $20 \mathrm{eV}$ will traverse a distance of 30 microns, in the absence of collisions. These values for current density and spot size are order-of-magnitude consistent with what is conventionally considered to be typical of cathode spots. As a reference, the typical lifetime of a cathode spot is thought to be microseconds to milliseconds, depending on the arc parameters [4]. The electron temperature required for the fit, $20 \mathrm{eV}$, is not unreasonable: the ionization potential for $1 i^{2+}$ the energy necessary to remove the third bound electron so as to create $\mathrm{Ti}^{3+}$ - is $25 \mathrm{eV}[45]$, and the cross-section for ionization does not become 
significant until the energy is around twice this value; thus electrons with energy several tens of ev are necessary if $\mathrm{Ti}^{3+}$ ions are to be seen in the spectrum, and assuming the ionization mechanism is indeed electron impact.

The gadolinium spectrum provides an illustration of the possible ambiguity in the fitting procedure that we are using here. Figure 26 shows the computed charge state evolution for a gadolinium plasma for two different sets of input parameters. Figure $26(\mathrm{a})$ is for a monoenergetic electron velocity distribution with $E_{e}=20 \mathrm{eV}$, a Gaussian distribution of plasma density, and with no steady injection of fresh neutrals; Figure 26(b) assumes a Maxwellian velocity distribution with $7_{\mathrm{e}}=3.5 \mathrm{eV}$, a uniform plasma density distribution, and steady injection of fresh neutrals. Each calculation provides a good fit to the experimental gadolinium spectrum of figure 16, the first for $j_{e^{\top} i}=5.6 \times 10^{16}$ electrons/cm ${ }^{2}$ and the second for $j_{e^{\tau} i}=5.2 \times 10^{17}$ electrons $/ \mathrm{cm}^{2}$. These are not the only possible fits either. Equally good fits can be made by substituting the steady injection of neutrals for a Gaussian radial density distribution and retaining all other parameters of Figure 26(a), or by using a monoenergetic elsctron distribution of energy $24 \mathrm{eV}$, a uniform spot density, and $j_{e^{\tau} j}=1.1 x$ $10^{16}$ electrons $/ \mathrm{cm}^{2}$, or by choosing other sets of parameters.

We've tried to avoid this ambiguity by fitting the average charge state for a number of different cathode materials to a single, consistent set of plasma parameters. This approach does rot in general provide a detailed fit to all of the individual CSO's, but it does provide a consistent model for prediction of the mean charge state, $\bar{Q}$. The plasma is taken to be as follows: the electron energy is assumed to be monoenergetic and equal to the measured arc voltage, and the ion confinement $t$ imes $\tau_{j}$ are scaled as the square root of the ion mass over the electron energy. We find that the best fit to the data is given either by choosing a Gaussian density distribution along with no steady injection of fresh neutrals, or by choosing a uniform radial density distribution along with steady injection of fresh neutrals; for the range of parameters considered, either choice fits the data. We consider the choice of steady injection of fresh neutrals to be inappropriate, however, because the ions are assumed to be moving through the cathode spot plasma and therefore moving away from the source of fresh neutral particles. Furthermore, a Gaussian density distribution of the spot plesma is likely. A Maxwellian electron energy distribution gives a considerably poorer fit to the data. 
In Figure 27 the experimentally measured values of $\bar{Q}$, as determined from the data of Table 1 , are plotted as a function of atomic number 2 . The values of $\bar{Q}$ predicted by the above model, for those cathode materials for which we have arc voltage data, are shown, and the fit to the data is excellent. Also plotted is a phenomenological fit to the data points, the function

$$
\overline{0}=0.72 z^{1 / 3}
$$

While this function does not fit the data as well as the model based on the measured arc voltages and the Lotz cross sections, it is useful as a first approximation, apart from the soft metals $S n$ and $P b$, and $U$. Soft cathode materials have a CSD with lower mean charge state than might be expected; the uranium data point might be high because the arc current used was high, perhaps over $400 \mathrm{~A}$. One can explore variations of $\bar{Q}$ with $T$, the melting point of the material, and fits can be found which add a little predictability to the phenomenological formula. For example a one-third power variation with melting point temperature helps, but the $f i t$ is not good over the entire range of $\mathrm{Z}$ and $\mathrm{T}$. 
Measurements have been made of the charge state distribution of the ions produced by the metal vapor vacuum arc for a wide range of cathode materials, both metallic elements and conducting compounds. Multiply charged ions are produced, the charge state distributions for which can be interpreted as being due to stepwise ionization by collisions within the cathode spot with the intense electron current density that concentrates at the spot. Consistent parameters of the cathode spot plasma that are implied by the model are: an electron current density of order 1 to $10 \mathrm{MA} / \mathrm{cm}^{2}$, and a spot of size of order tens of microns and with a Gaussian density distribution; the electron energy is taken as equal to the arc yoltage, scme tens of electron volts. The mean charge state $\overline{0}$ increases with the $z$ of the cathode element, and an approximate phencmenological fit to the data is provided by the formula $\bar{Q}=$ $0.722^{1 / 3}$. On top of this variation with atomic number there is also a trend in which soft, low melting point, materials have lower mean charge state than predicted by the formula. The inean charge state can be predicted well from the model, using the measured value of arc voltage.

These results are important fundamentally because they add to the pool of knowledge about cathode spot behavior, a plasma phenomenon still far from understood. The MEVVA ion source has demonstrated itself as being a suitable tool for investigation of the physics of metal vapor vacuum arcs. Finally, the data provide the MEVVA ion source user with practical information on source perforinance. 


\section{ACKNOWLEDGMENTS}

We are greatly indebted to Bob MacGill and Bob Wright for the mechanical

design and fabrication of the ion source, and for their continued invaluable contributions throughout the experimental program. 
1. R. W. Sorensen and H. E. Mendenhall, Trans. AIEE, Pt. III, 45, 1102 (1926).

2. J. D. Cobine, Elect. Eng. 81, 13 (1962).

3. J. M. Lafferty, editor, "Vacuum Arcs - Theory and Application", John Wi iey and Sons, New York, 1980.

4. G. A. Lyubimov and V. I. Rakhovskii, Sov. Phys. Usp. 21, 693 (1978).

5. A. A. Plyutto, V. N. Ryzhkov and A. T. Kapin, Sov. Phys. JElP 20, 328 (1965).

6. W. D. Davis and H. C. Miller, J. Appl. Phys. 40, 2212 (1969).

7. C. W. Kimblin, Proc. IEEE 59, 546 (1971).

8. A. S. Gilmour, Jr., and D. L. Lockwood, Proc. IEEE 60, 977 (1972).

9. C. W. Kimblin, J. Appl. Phys. 44, 3074 (1973).

10. J. E. Daalder, J. Phys. D: Appl. Phys. ․, 130 (1975).

11. J. W. Robinson and M. Ham, IEEE Trans. Plasma Sci. PS-3, 222 (1975).

12. J. E. Daalder J. Phys. D: App 1. Phys. 9, 2379 (1976).

13. E. Hantzsche, Proceedings of the 13th International Conference on Phenomena in Ionized Gases (Physical Society, GDR, Berlin, 1977), Vol. 3, p. 121.

14. V. Hermoch, ibid, p. 677.

15. D. T. Tuma, C. L. Chen and D. K. Davies, J. App1. Phys. 49, 3821 (1978).

16. J. E. Daalder, Physica 104C. 91 (1981).

17. IEEE Irans. Plasma Sci. PS-11. No. 3 (1983). Special issue on vacuum discharge plasmas.

18. IEEE Irans. Plasma Sci. PS-13, No. 5 (1985). Special issue on vacuum discharge plasmas.

19. "Electromagnetic Separation of 1sotopes in Commercial Quantities", edited by R. K. Wakerling and A. Guthrie (McGraw Hill, New York, 1951), p. 324.

20. E. I. Revutskit, G. M. Skoromnyi, Yu. F. Kulygin and I. I. Goncharenko, in Proceedings of the Soviet Conference on Charged Particle Accelerators, Moscow, 9-16 October 1968, edited by A. A. Vasilev (USAEC), Vol, 1, p. 447.

21. R. J. Adler and S. T. Picraux, Nucl. Instrum. and Methods B6, 123 (1985).

22. S. Humphries, Jr., M. Savage and D. M. Woodal1, Appl. Phys. Lett. 47, 468 (1985).

23. C. Burkhart, S. Coffey, G. Cooper, S. Humphries, Jr., L. K. Len, A. D. Logan, M. Savage and D. M. Wooda 11, Nuc 1. Instrum. and Methods B10, 192 (1985). 
24. S. Humphries, Jr., C. Burkart, S. Coffey, G. Cooper, L. K. Len, M. Savage, D. M. Woodall, H. Rutkowski, H, Oona and R. Shurter, J. Appl. Phys. 59, 1790 (1986).

25. I. G. Brown, J. E. Galvin and R. A. MacGill, Appl. Phys. Lett. 47, 358 (1985).

26. 1. G. Brown, IEEE Trans. Nuc 1. Sci. NS-32, 1723 (1985).

27. I. G. Brown, J. E. Galvin, B. F. Gavin and R. A. MacGill, Rev. Sci. Instrum. 57, 1069 (1986).

28. I. G. Brown, J. E. Galvin, R. Keller, P. Spaedtke, R. W. Mueller and J. Bolle, Nucl. Instrum, and Methods A245, 217 (1986).

29. V. M. Lunev, V. G. Padalka and V. M. Khoroshikh, Sov. Phys. Tech. Phys. 22, $858(1977)$.

30. H. C. Miller, J. Phys. D: App1. Phys. 12, 1293 (1979).

31. H. C. Miller, J. Appl. Phys. 52,4523 (1981).

32. L. K. Len, C. Burkhart, G. W. Cooper, S. Humphries, Jr., M. Savage and D. M. Woodal1, IEEE Trans. Plasma Sci. NS-14, 256 (1986).

33. J. E. Galvin and I. G. Brown, Rev. Sci. Instr. 55, 1866 (1984).

34. J. E. Galvin and I. G. Brown, Rev. Sci. Instr. 56. 1972 (1985).

35. R. Keller, GSI 1980 Annual Report, GSI Report 81-2, p. 263.

36. B. L. Schram, Physica 32, 197 (1966).

37. A. Mueller. Physics Letters 113A, 415 (1986).

38. A. Mueller, C. Achenbach, E. Salzborn and R. Becker, J. Phys. B, 17, 1427 (1984).

39. H. Winter and B. H. Wolf, in Proceedings of the Second Symposium on Ion Sources and Formation of Ion Beams, Berkeley, California, October 1974; Lawrence Berkeley Laboratory Report LBL-3399, paper V-1.

40. H. Winter, in "Experimental Methods in Heavy Ion Physics". Lecture Notes in Physics Series, edited by $K$. Bethge (Springer, Berlin, 1978), p. 14.

41. SFEC Group, Report SFEC 110 - Cryebis II, Laboratoire National Saturne, Saclay, France, 1981.

42. W. Lotz, Z. Phys. 216, 241 (1968).

43. W. Lotz, J. Opt. Soc. Am. 57, 873 (1967).

44. W. Lotz, J. Opt. Soc. Am. 58, 915 (1968).

45. T. A. Carlson, C. W. Nestor, Jr., N. Wasserman and J. D. Mc Dowe 11, Atomic Data 2, 63 (1970).

46. T. A. Carlson and B. P. Pullen, Oak Ridge National Laboratory Report ORNL 4393 (1969). 
47. A. Mueller, E. Salzborn, Phys. lett. 62A, 391 (1977).

48. E. D. Donets, IEEE Trans. Nucl. Sci. NS-23, 897 (1976).

49. J. Arianer and C. Goldstein, IEEE Trans. Nuc 1. Sci. NS-23, 1979 (1976).

50. R. Becker and H. Klein, IEEE Trans. Nucl. Sci. NS-23, 1017 (1976).

51. R. W. Hamm, Los Alamos Scientific Laboratory report LA-7077-T (1977).

52. Proceedings of the Workshop on EBIS and Related Topics, June 15-16, 1977, Darmstadt, Germany, edited by B. H. Wolf and H. Klein, (GSI, Darmstadt).

53. 1. G. Brown and B. Feinberg, Nucl. Instr. and Meth. 220, 251 (1984).

54. V. O. Kostroun, Nucl. Instr. and Meth. B10/11, 771 (1985).

55. E. D. Donets and V. P. Ovsyannikov, Sov. Phys. JETP $\underline{53}, 466$ (1981). 


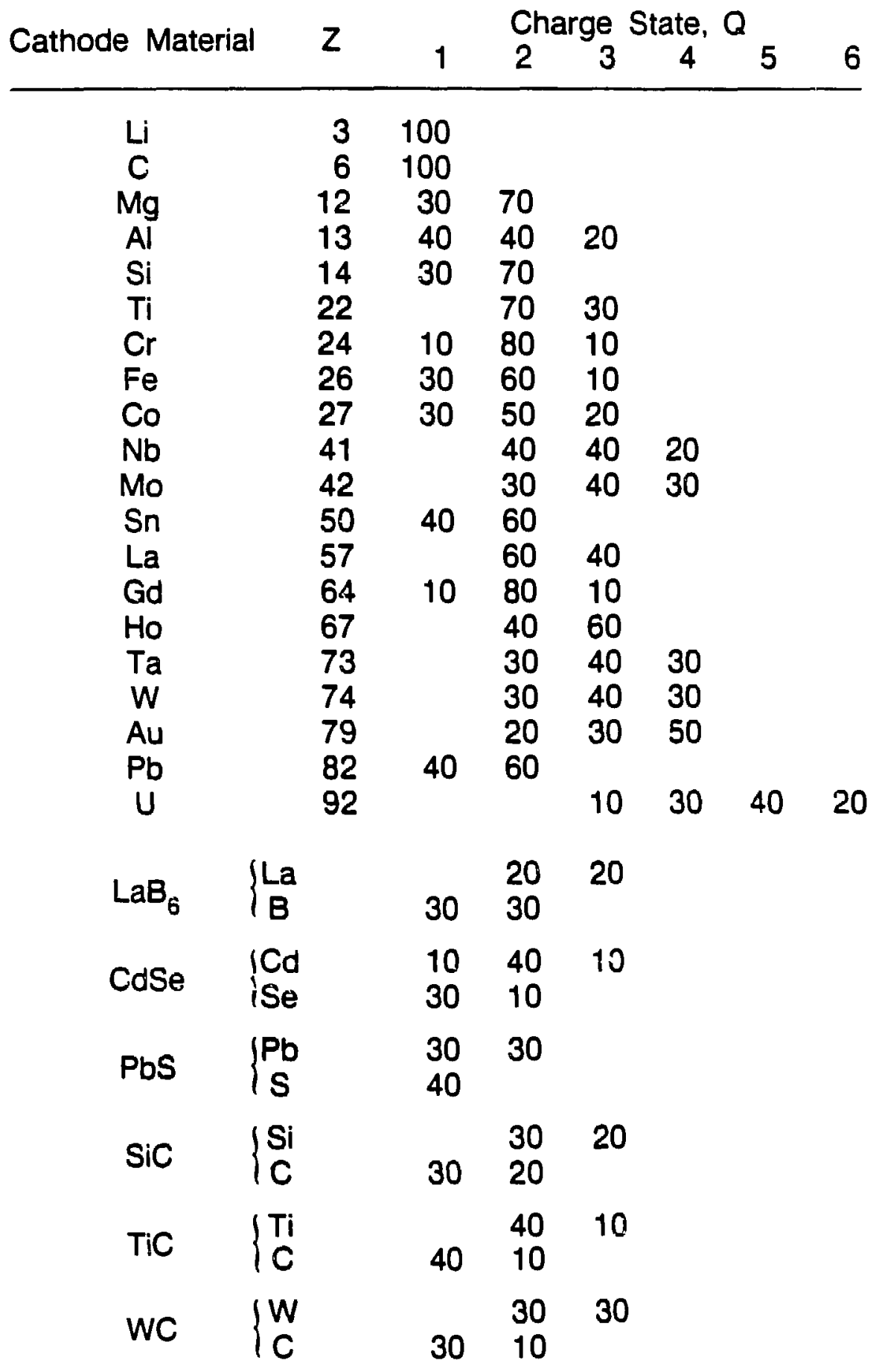

XBL 8610-4072

Table 1 Approximate charge state distributions for the complete range of elemental and compound cathode materials used. 


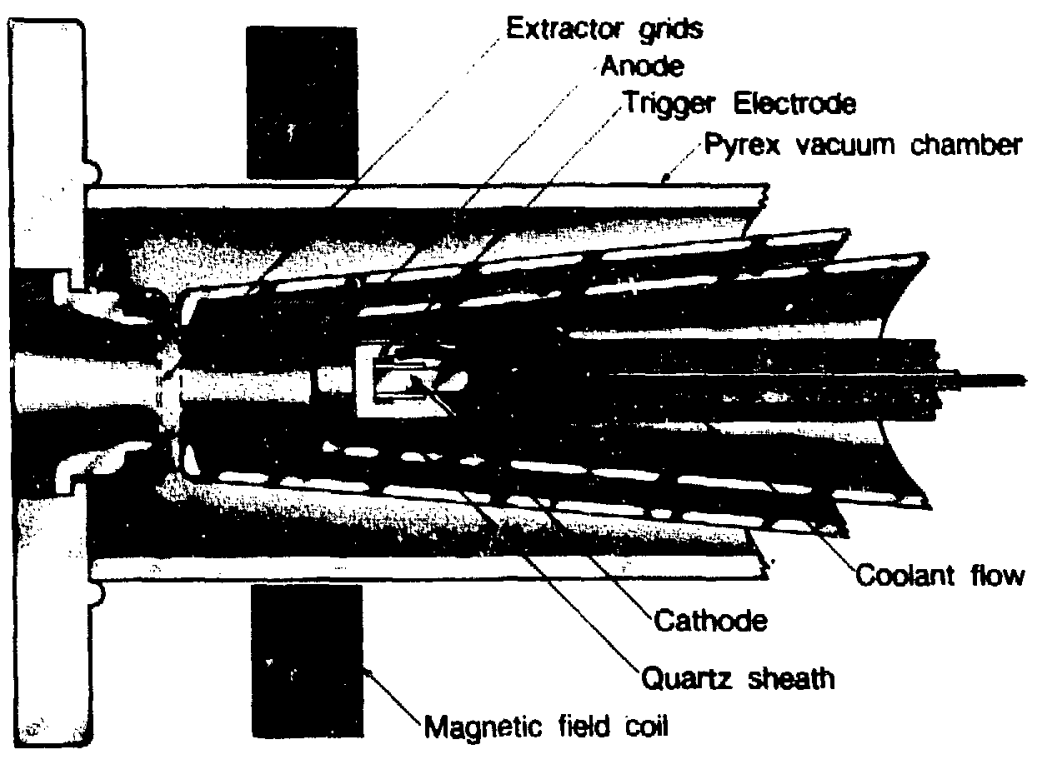

CBB $862-1326$

Fig. 1 Outline of the MEVVA II ion source. 


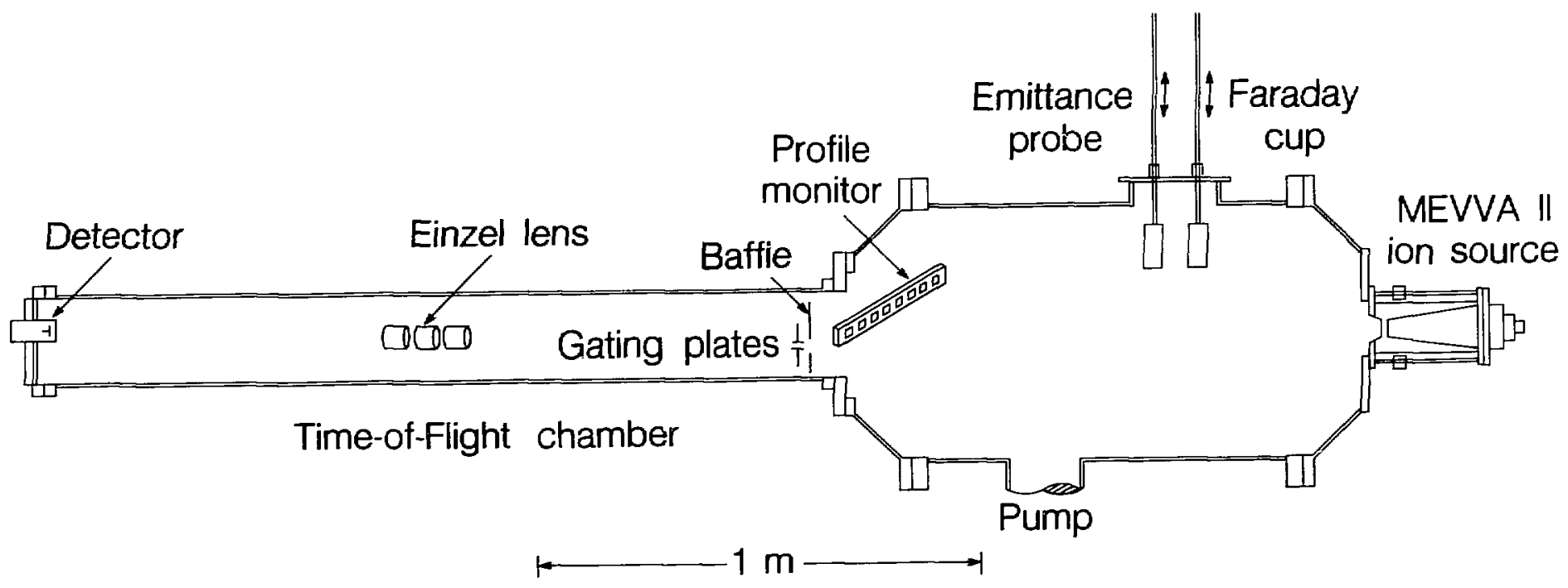

XBL 8610-9658

Fig. 2 Schematic of the experimental configuration. 


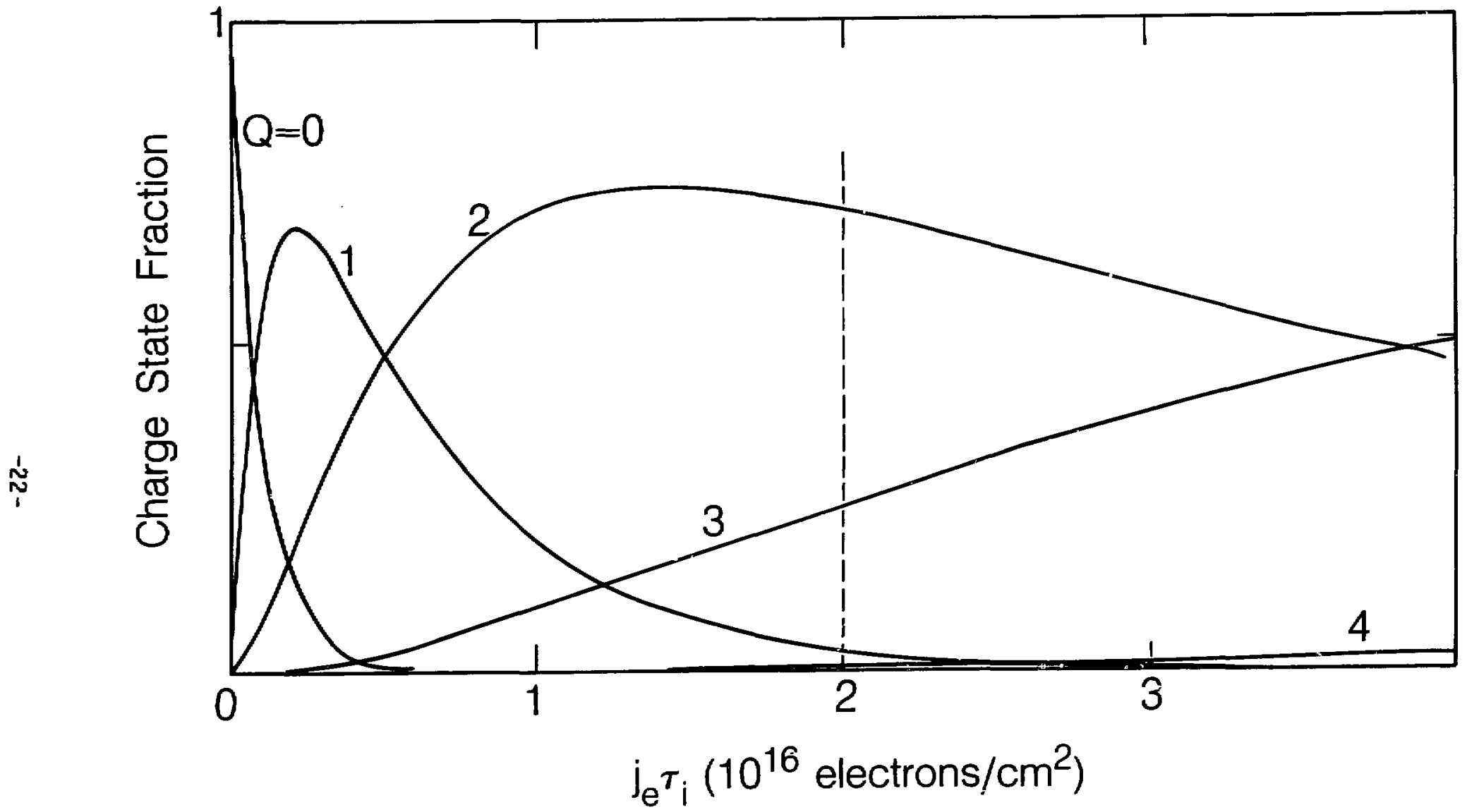

XBL 8610-9655

Fig. 3 Charge state distribution predicted by the stepwise ionization model. Titanium plasma, Maxwellian electron velactly distribution with $T_{e}=$ $20 \mathrm{eV}$; no recombination or charge exchange. 


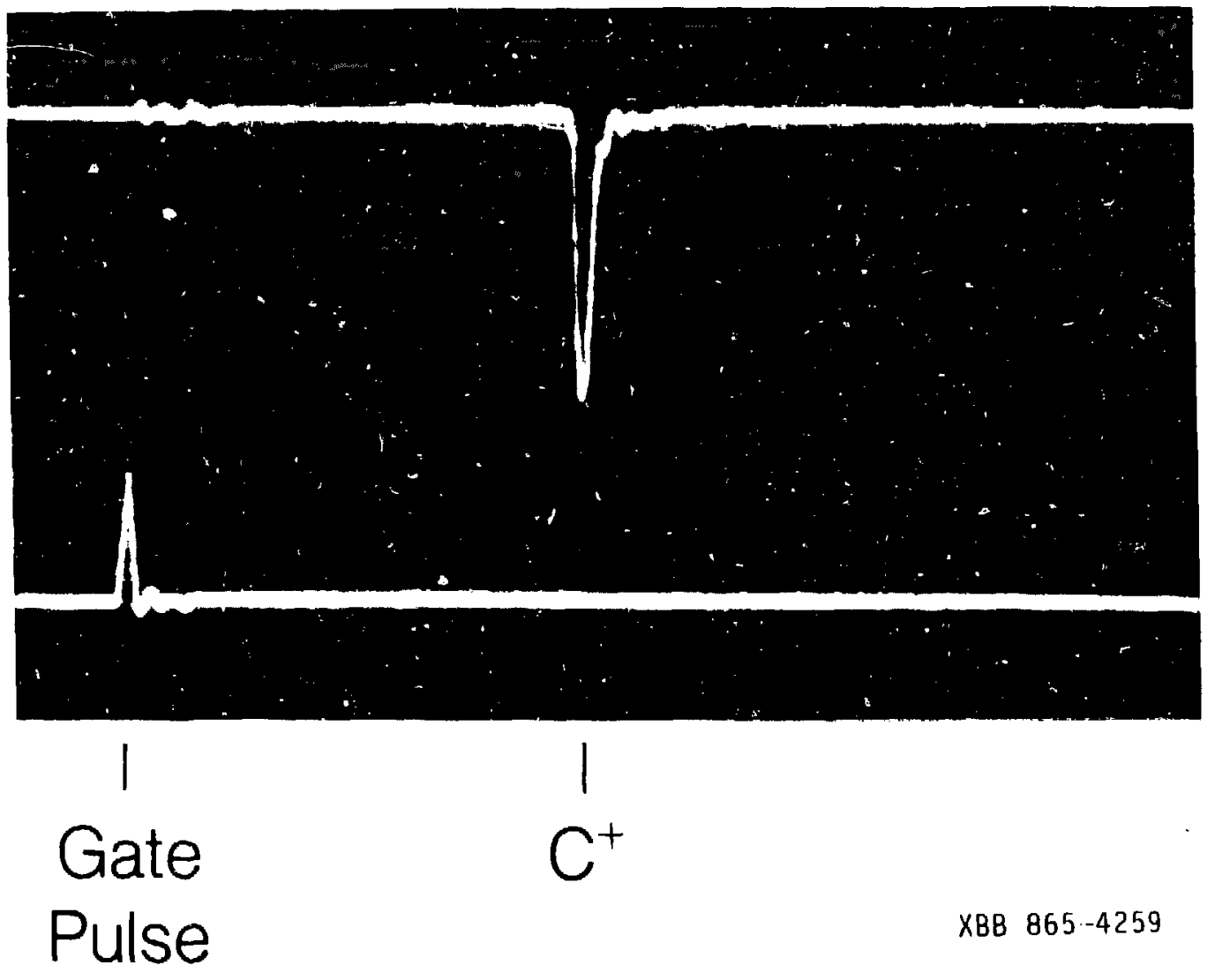

Fig. 4 Time-of-flight spectrum for carbon 


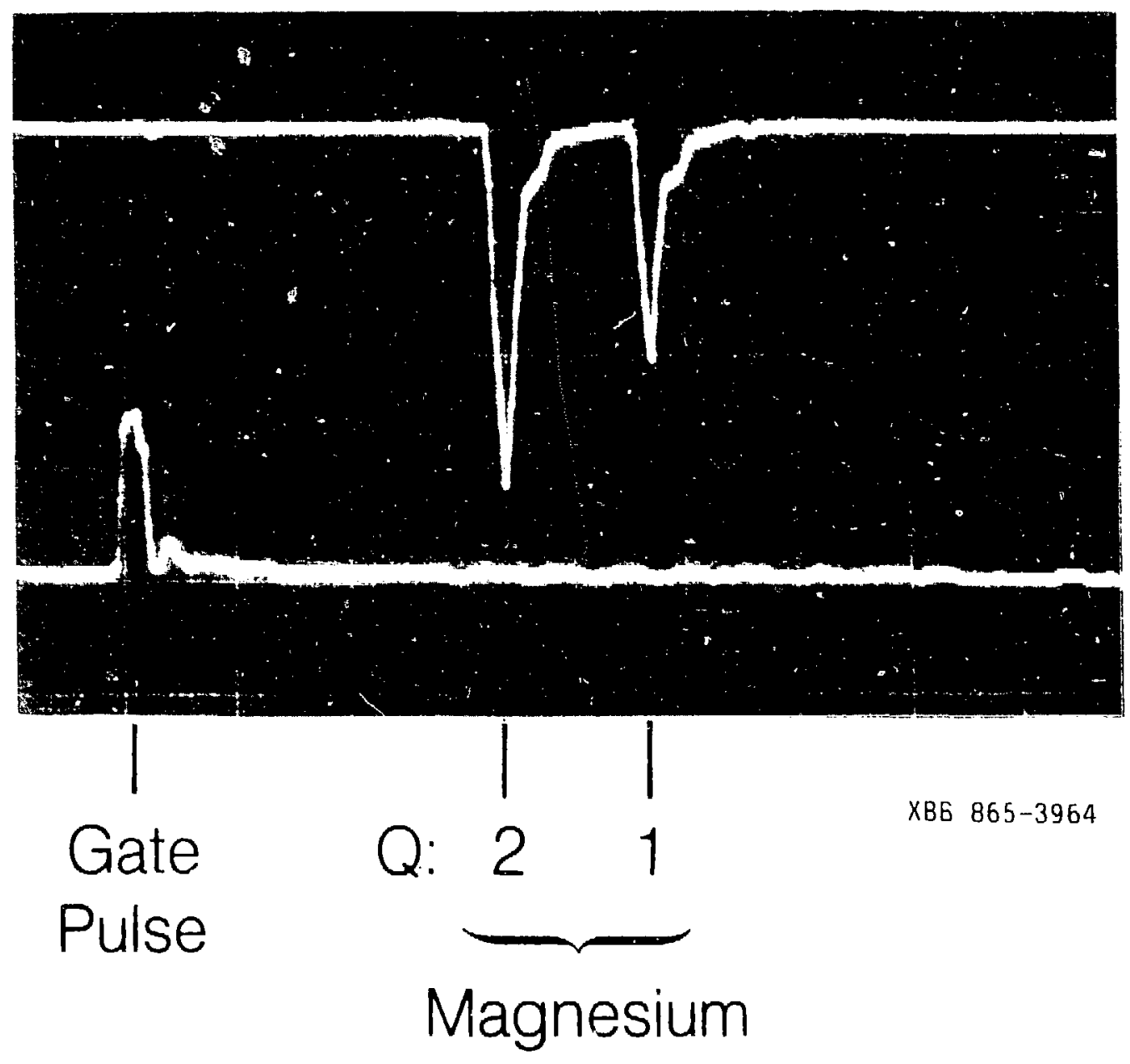

Fig. 5 lof spectrum for magnesium 


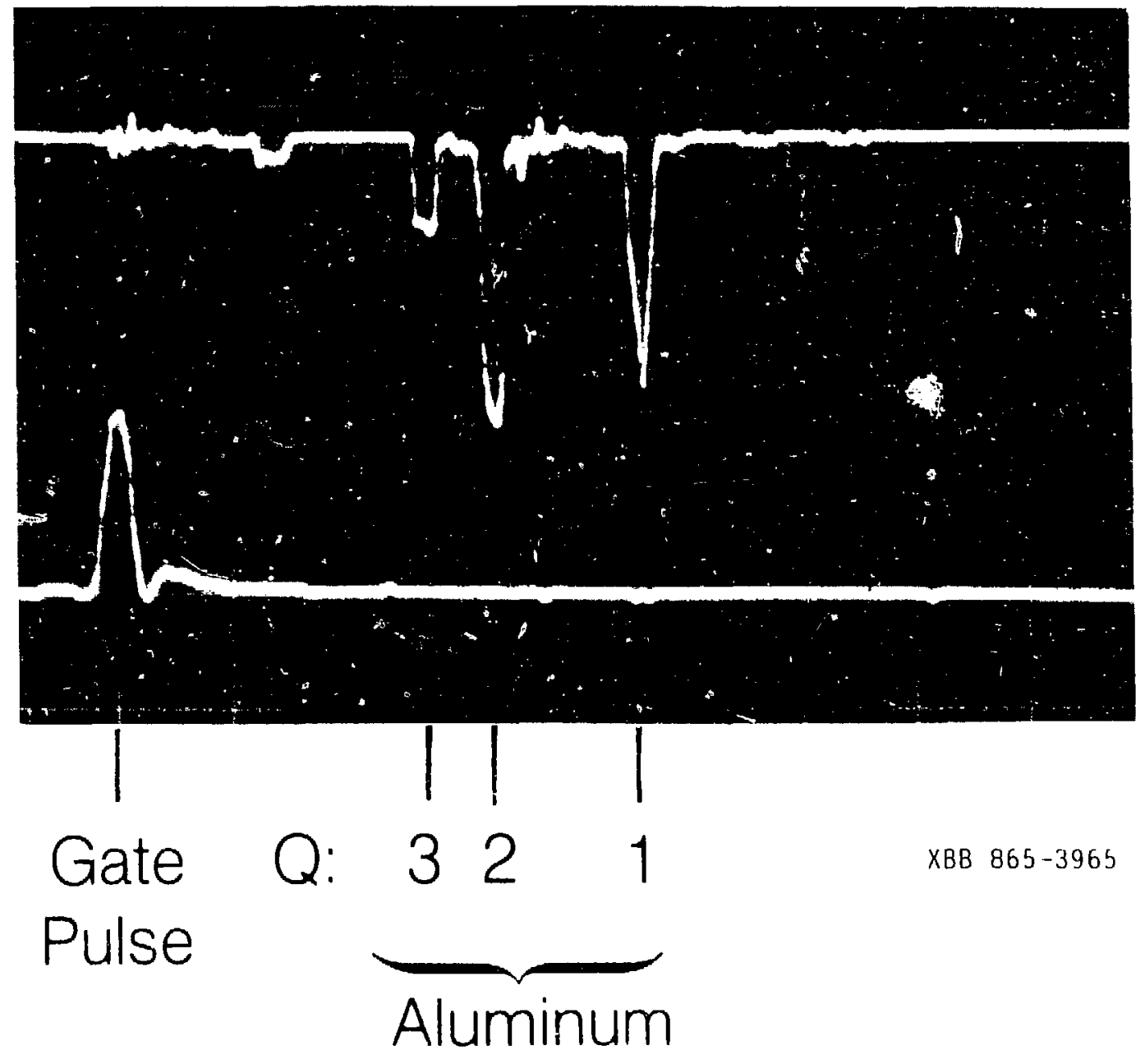

fig. 6 lof spectrum for aluminum 


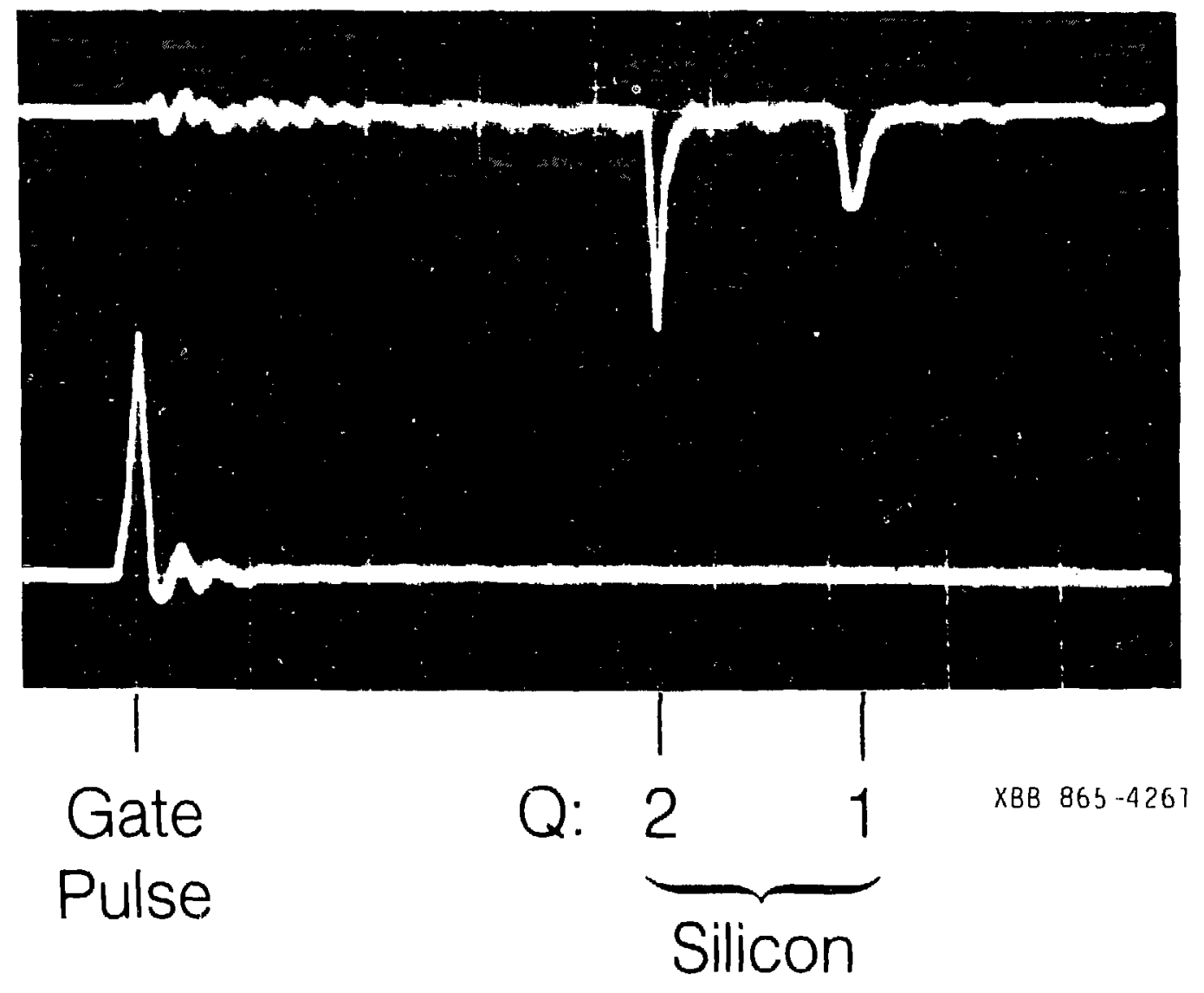

Fig. I Tot spectrum for silicon 


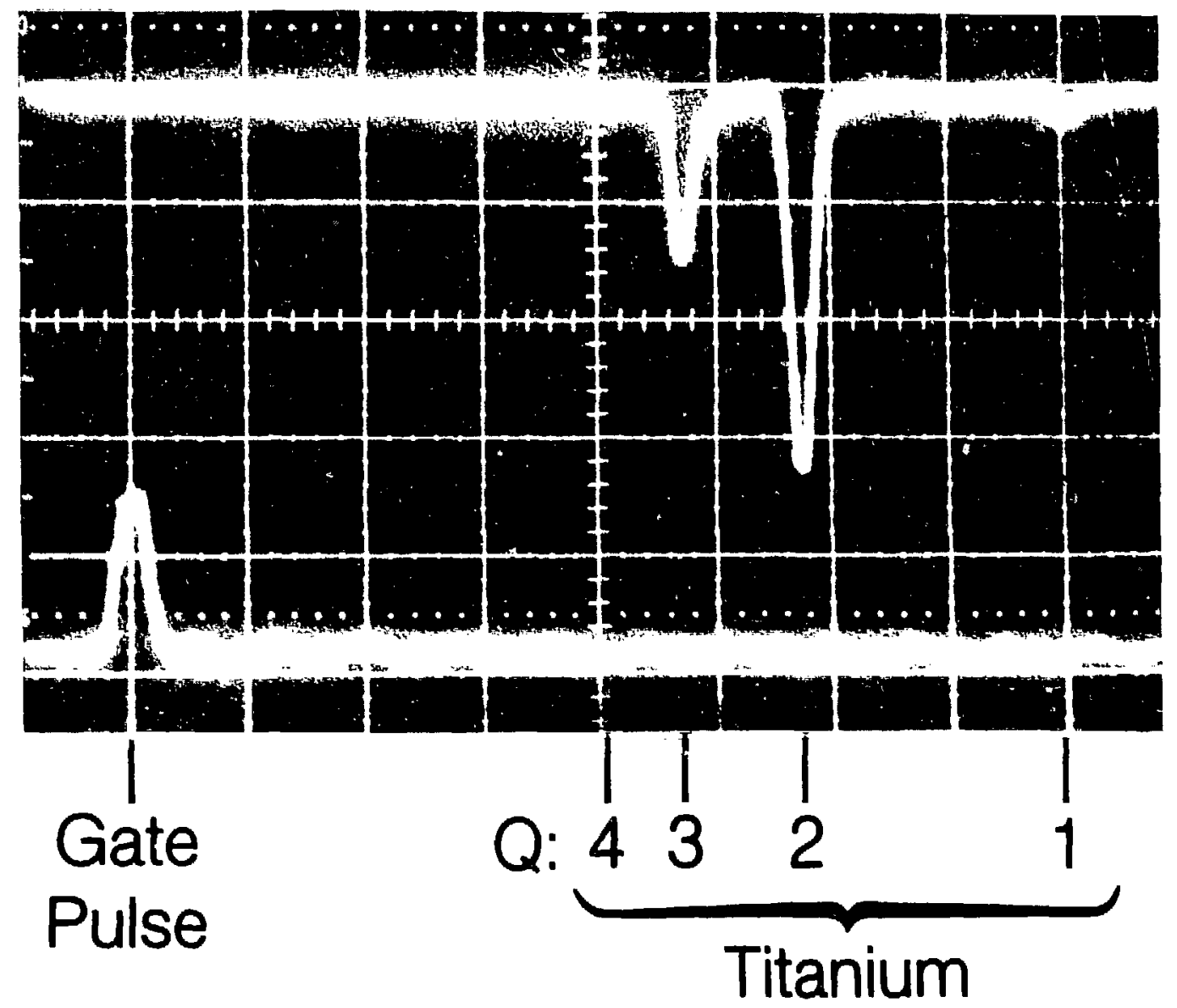

XBB $854-3256$

Fig. 8 TOF spectrum for titanium 


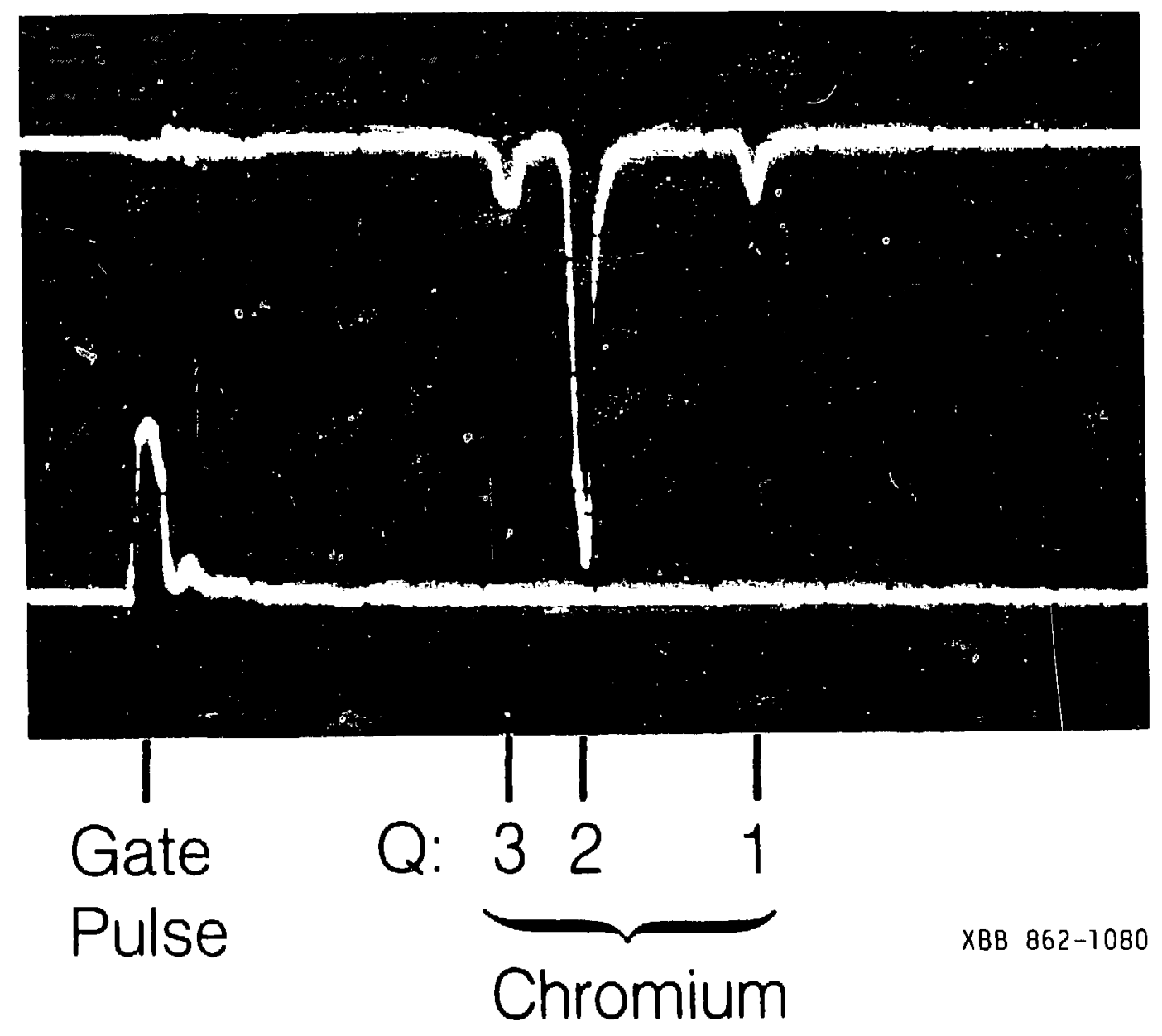

Fig. 9 TOF spectrum for chromium 


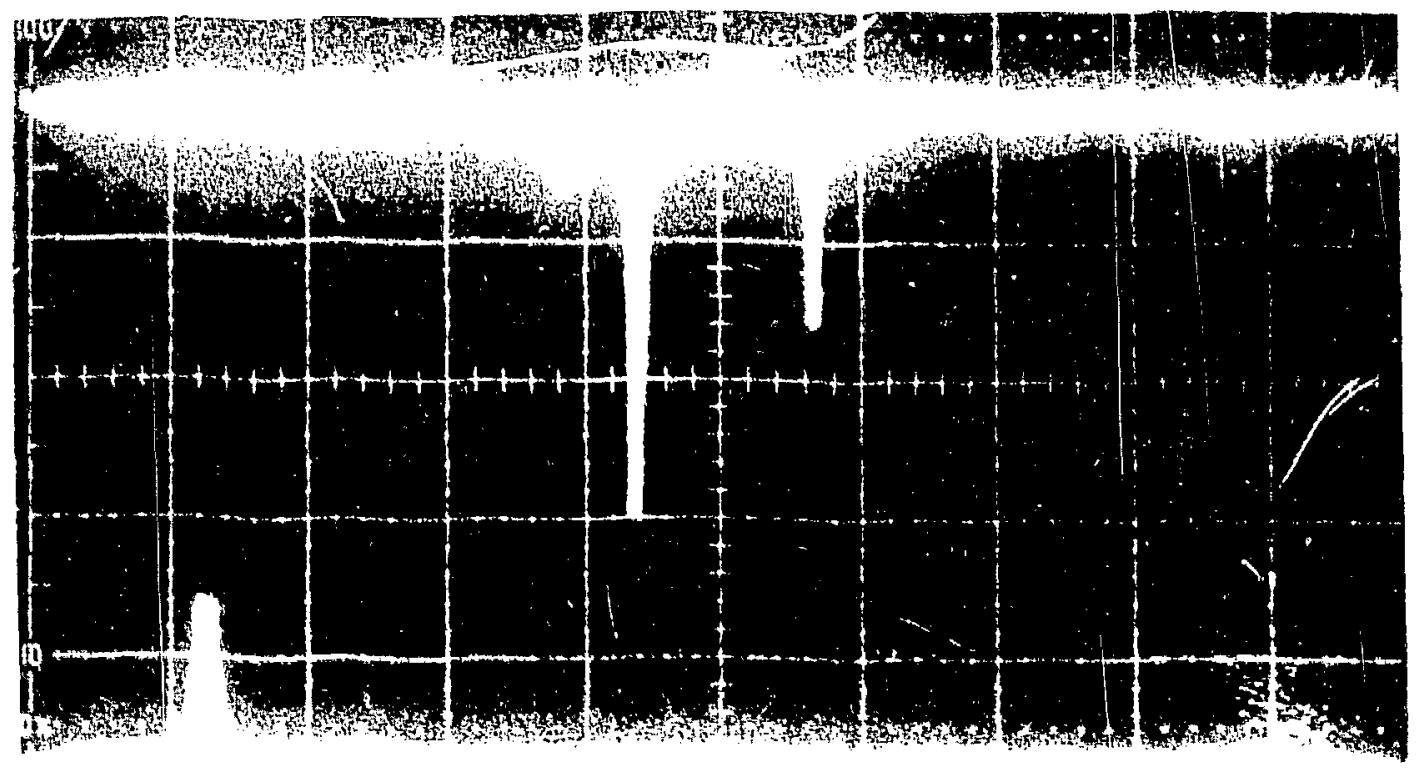

Hothom

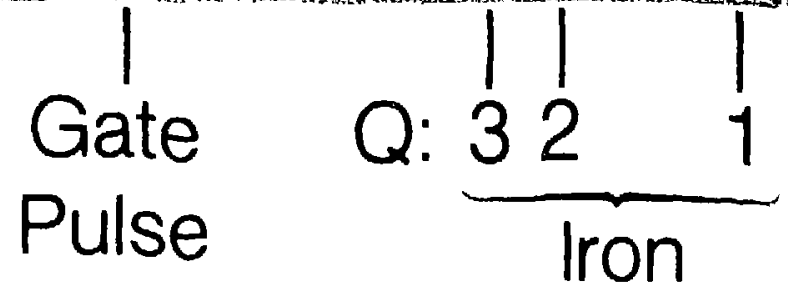

XBB $854-3254$

Fig. 10 TOF spectrum for iron 


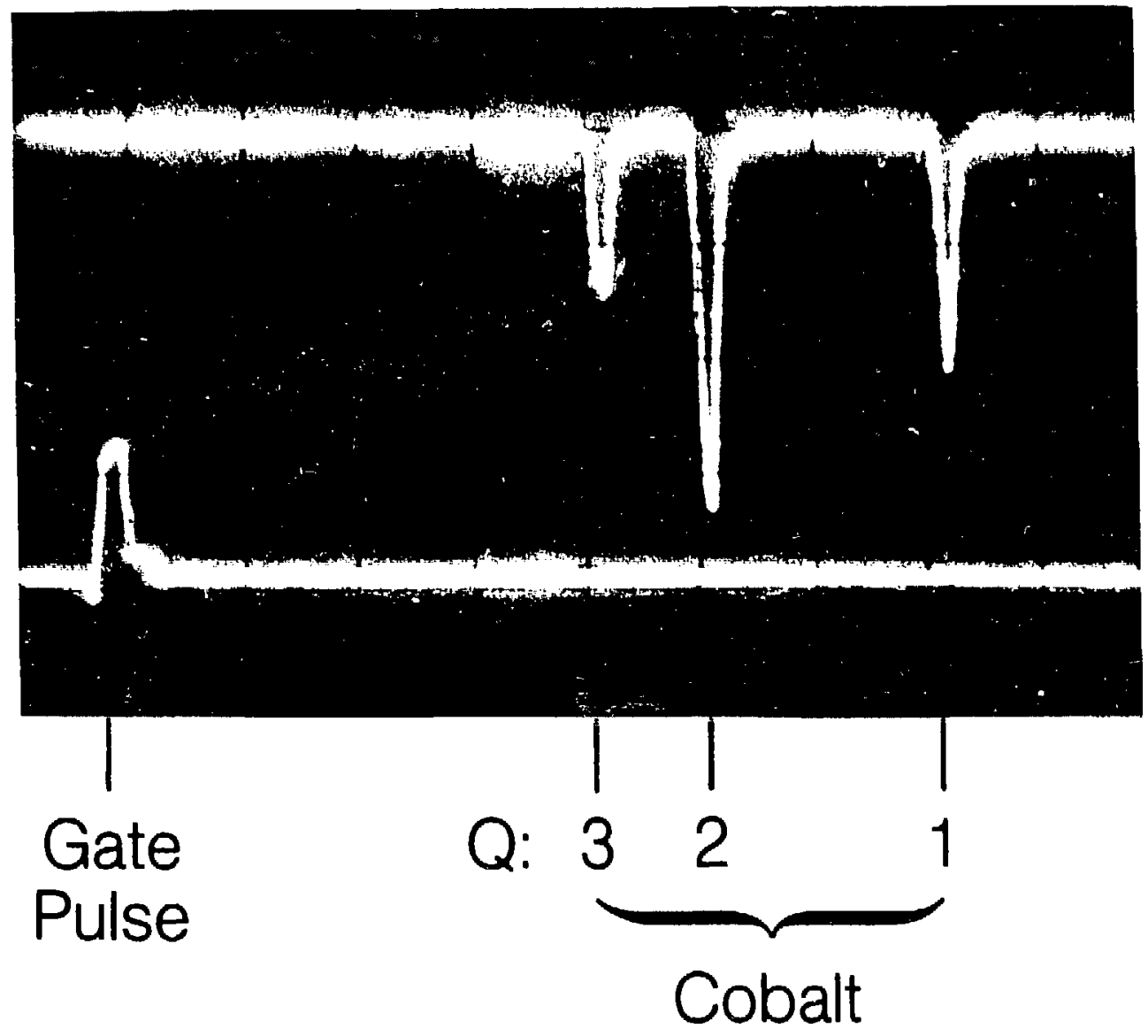

XBB $861-5715$

Fig. 11 TOF spectrum for cobalt 


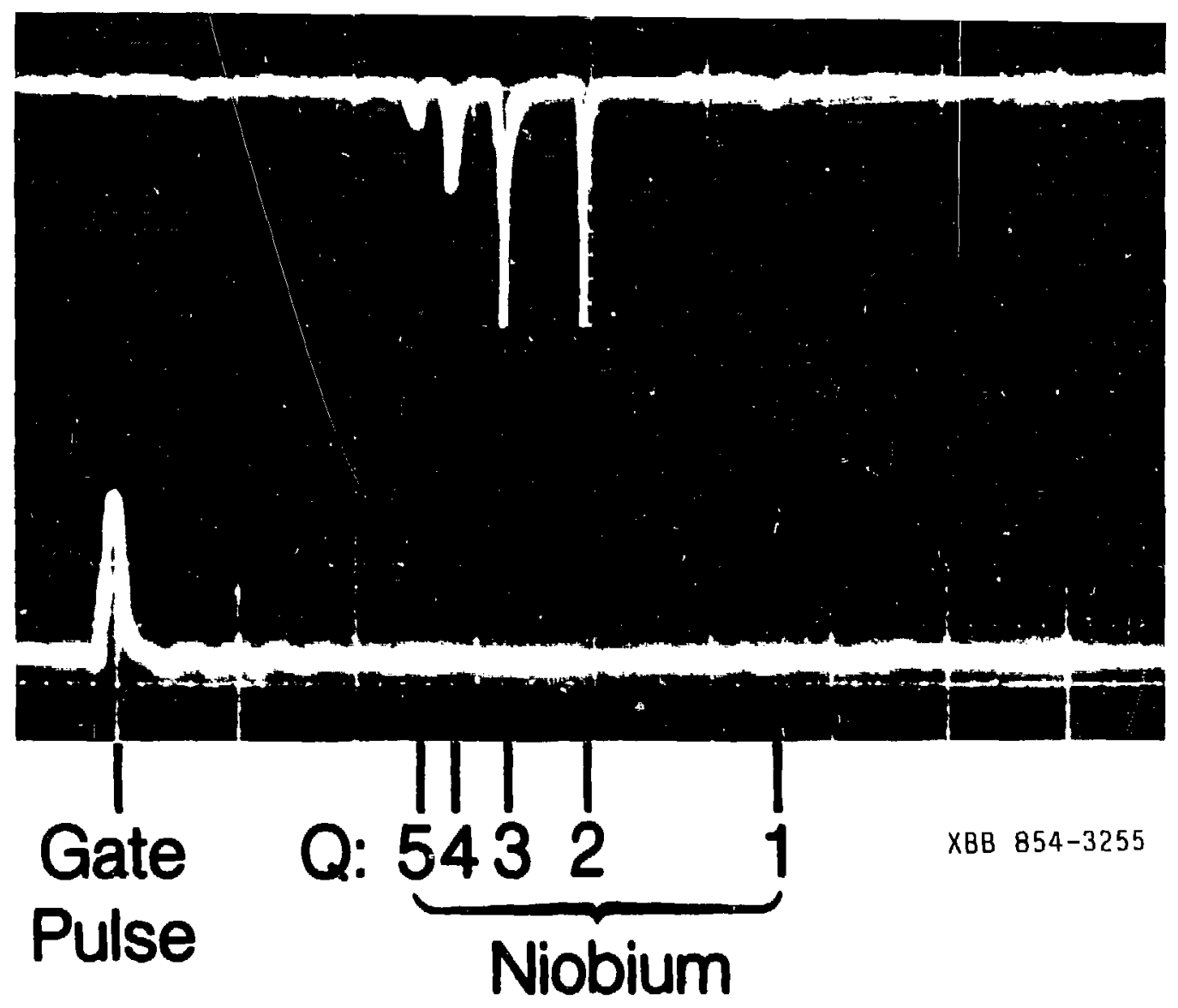

Fig. 12 TOF spectrum for niobium 


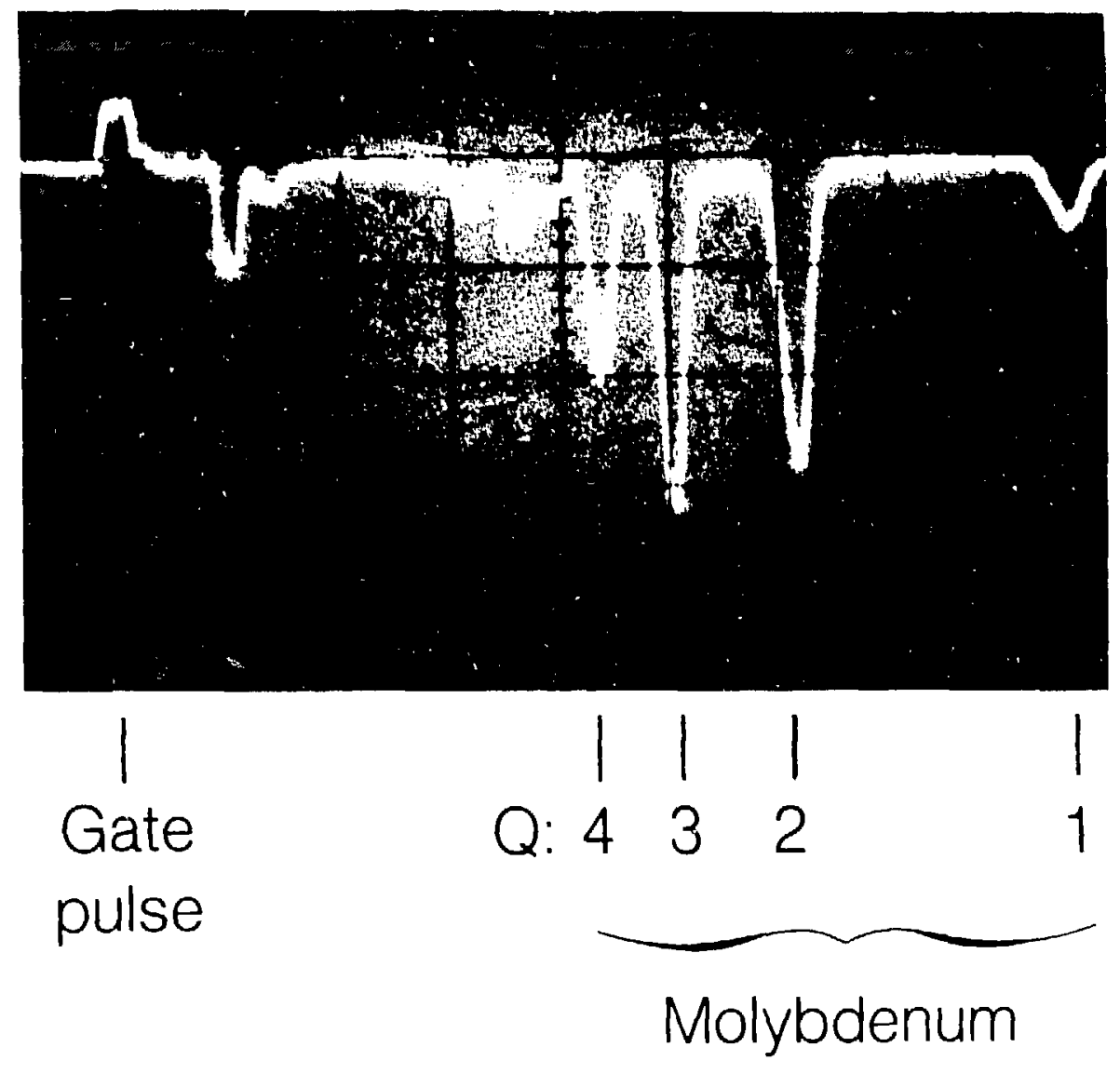

XBB $866-5353$

Fig. 13 TOF spectrum for molybdenum 


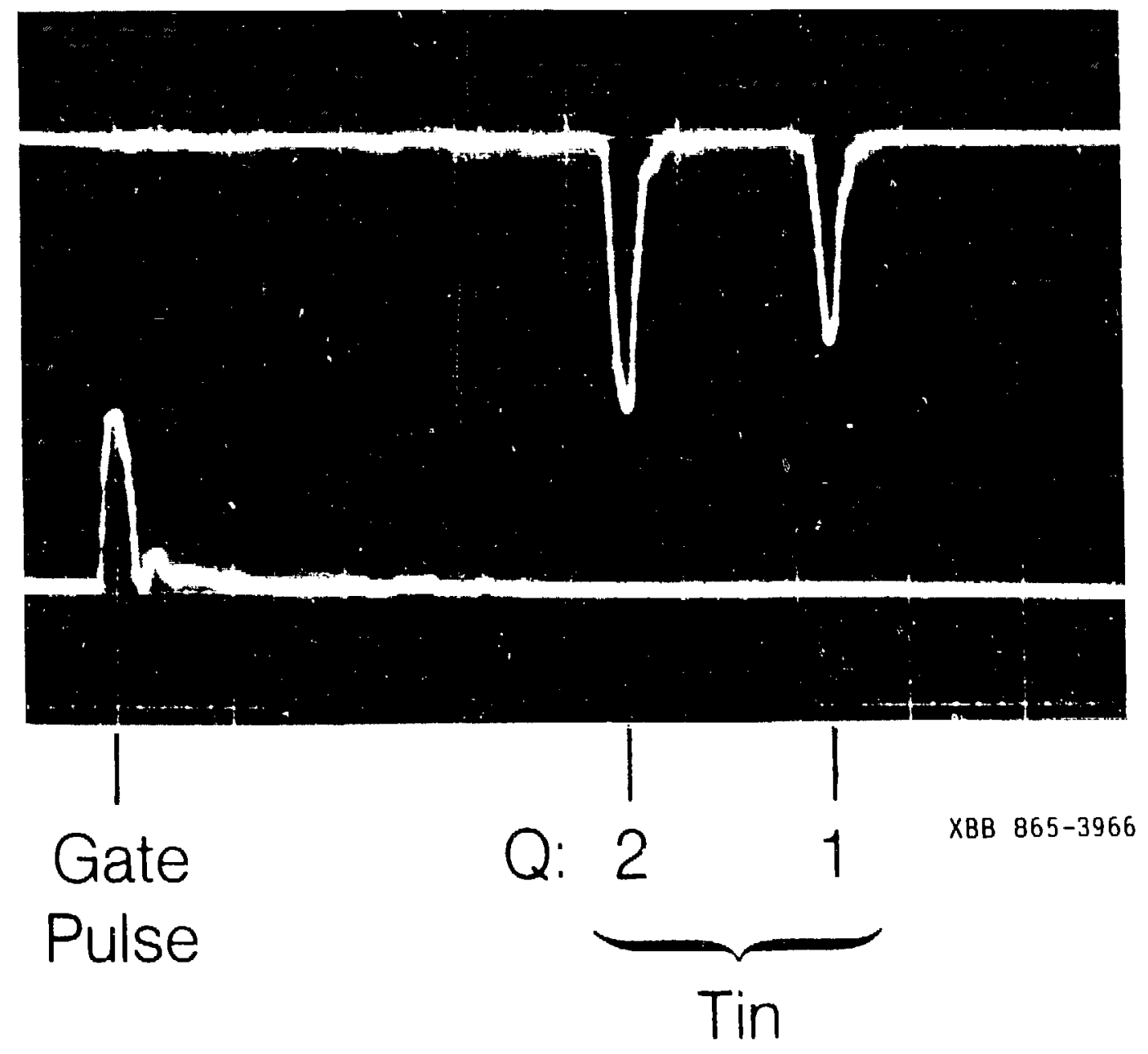

Fig. 14 TOF spectrum for tin 


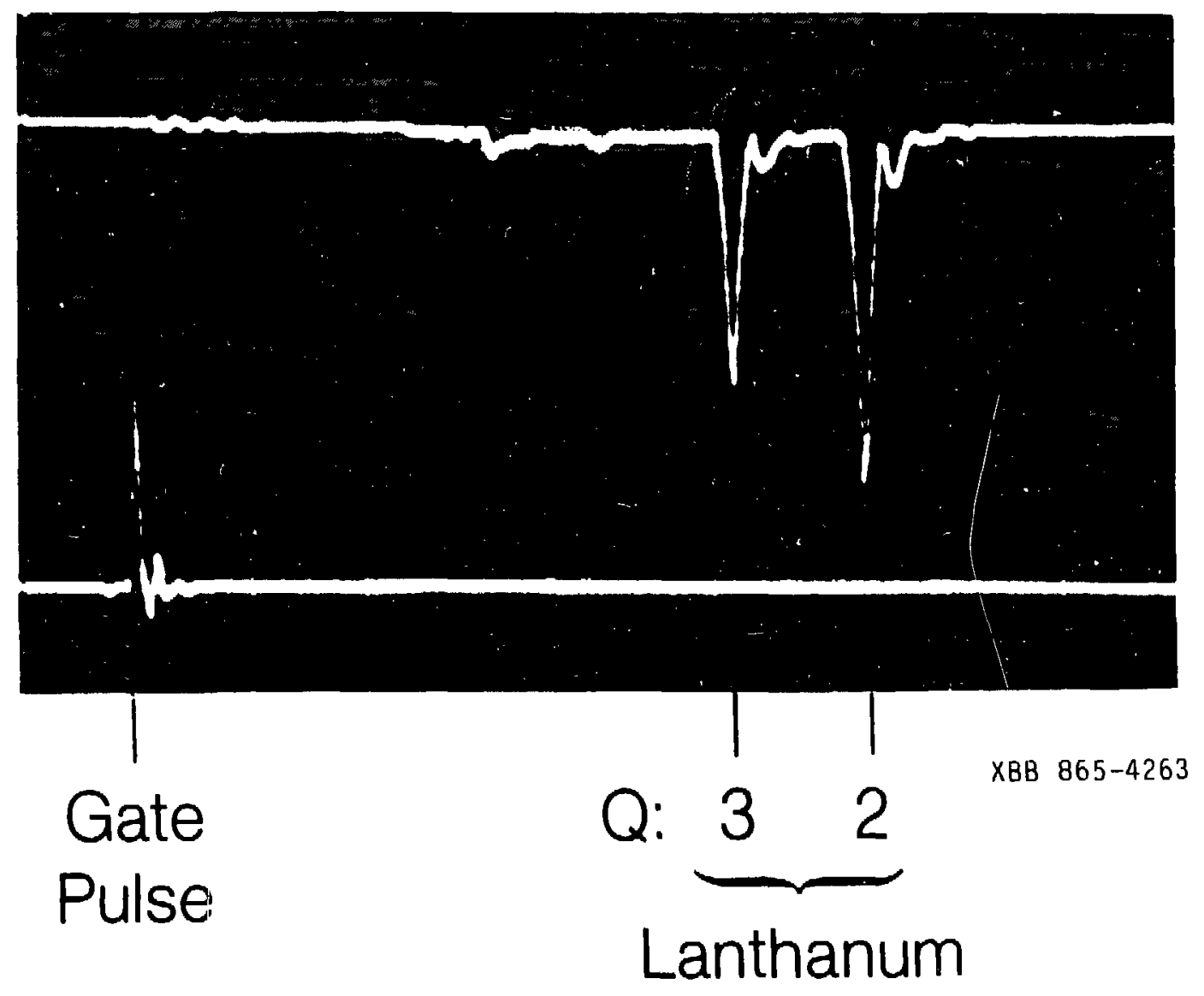

Fig. 15 TOF spectrum for lanthanum 


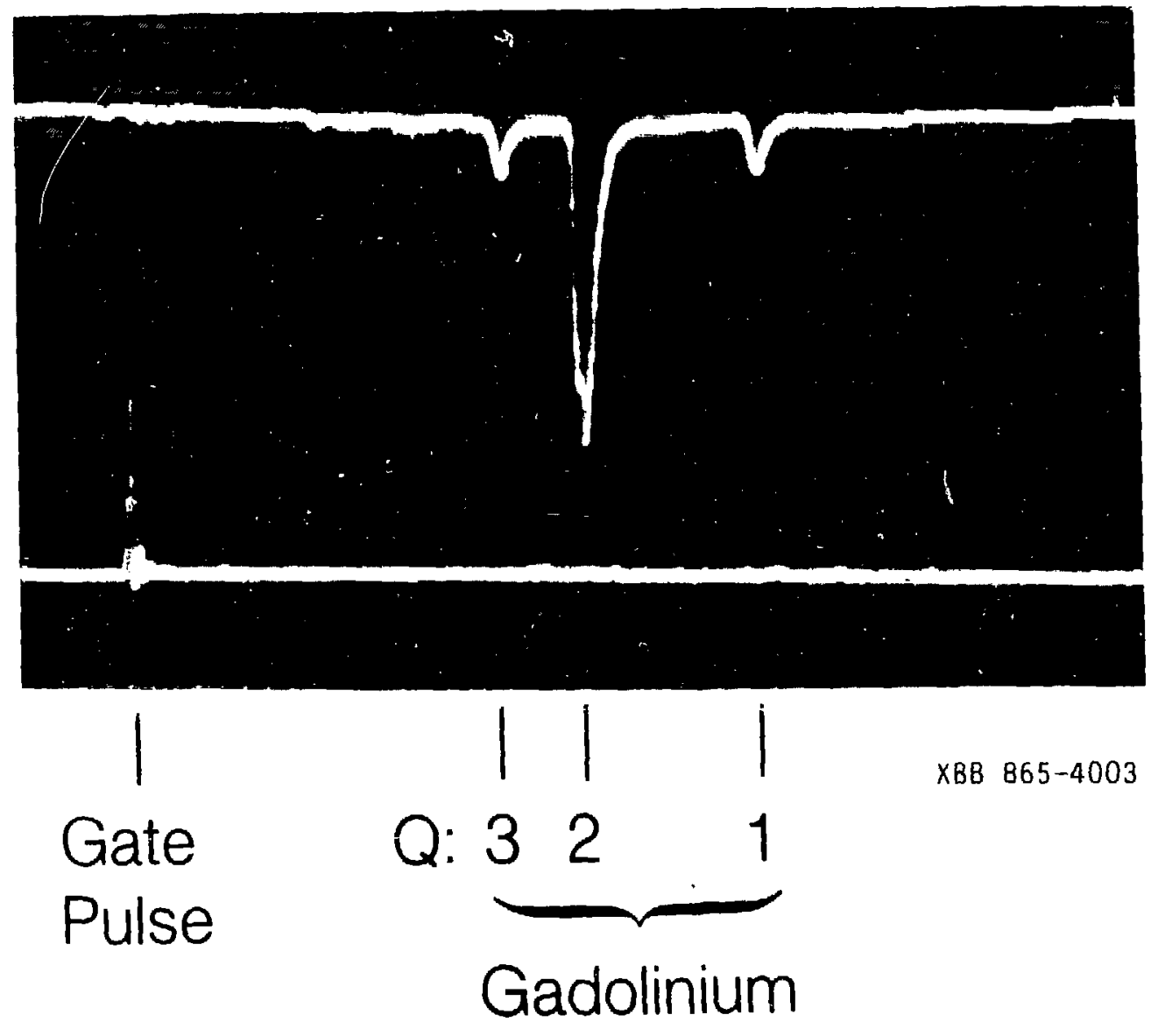

Fig. 16 TOF spectrum for gadolinium 


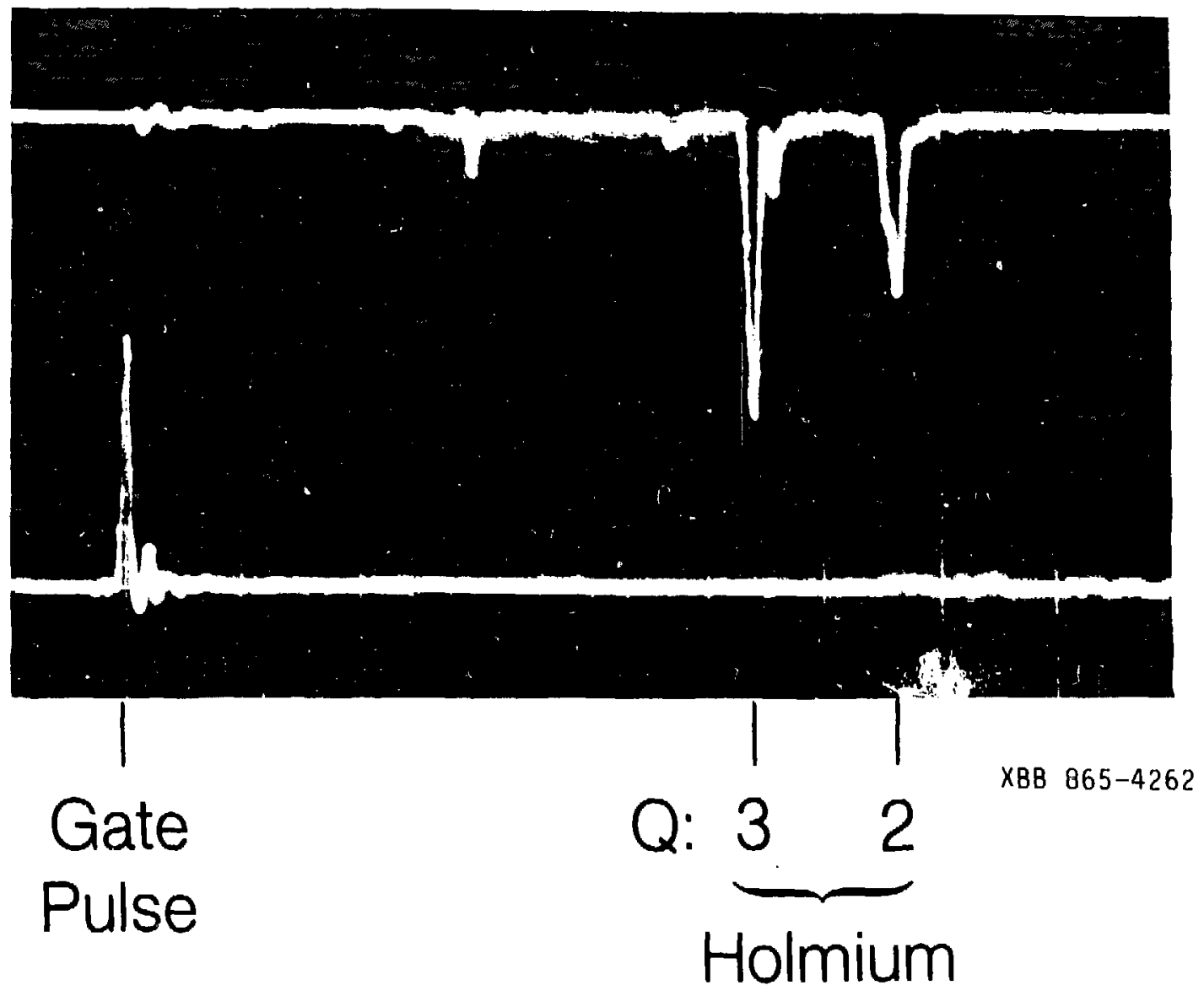

Fig. 17 TOF spectrum for holmium 


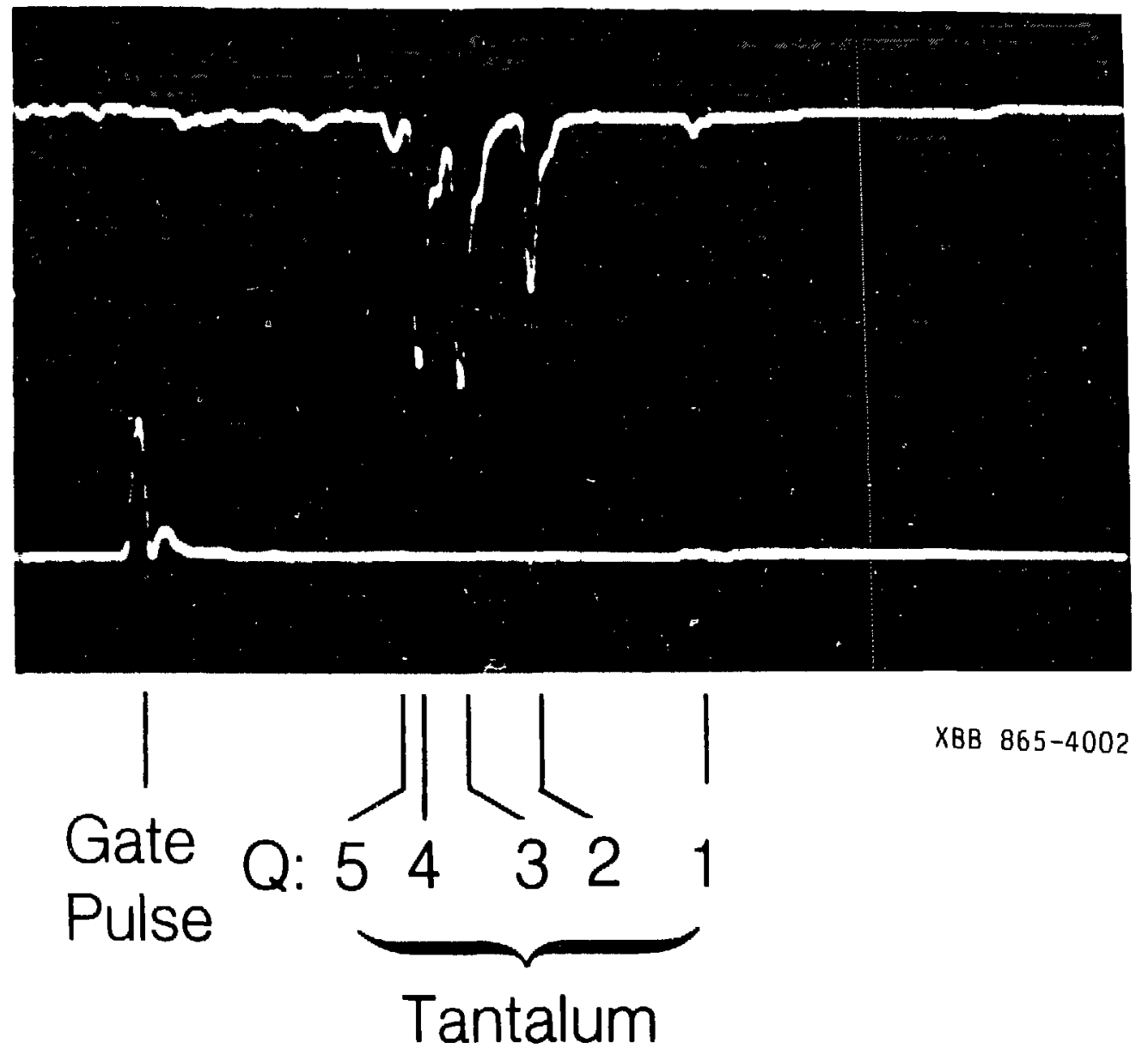

Fig. 18 TOF spectrum for tantalum 


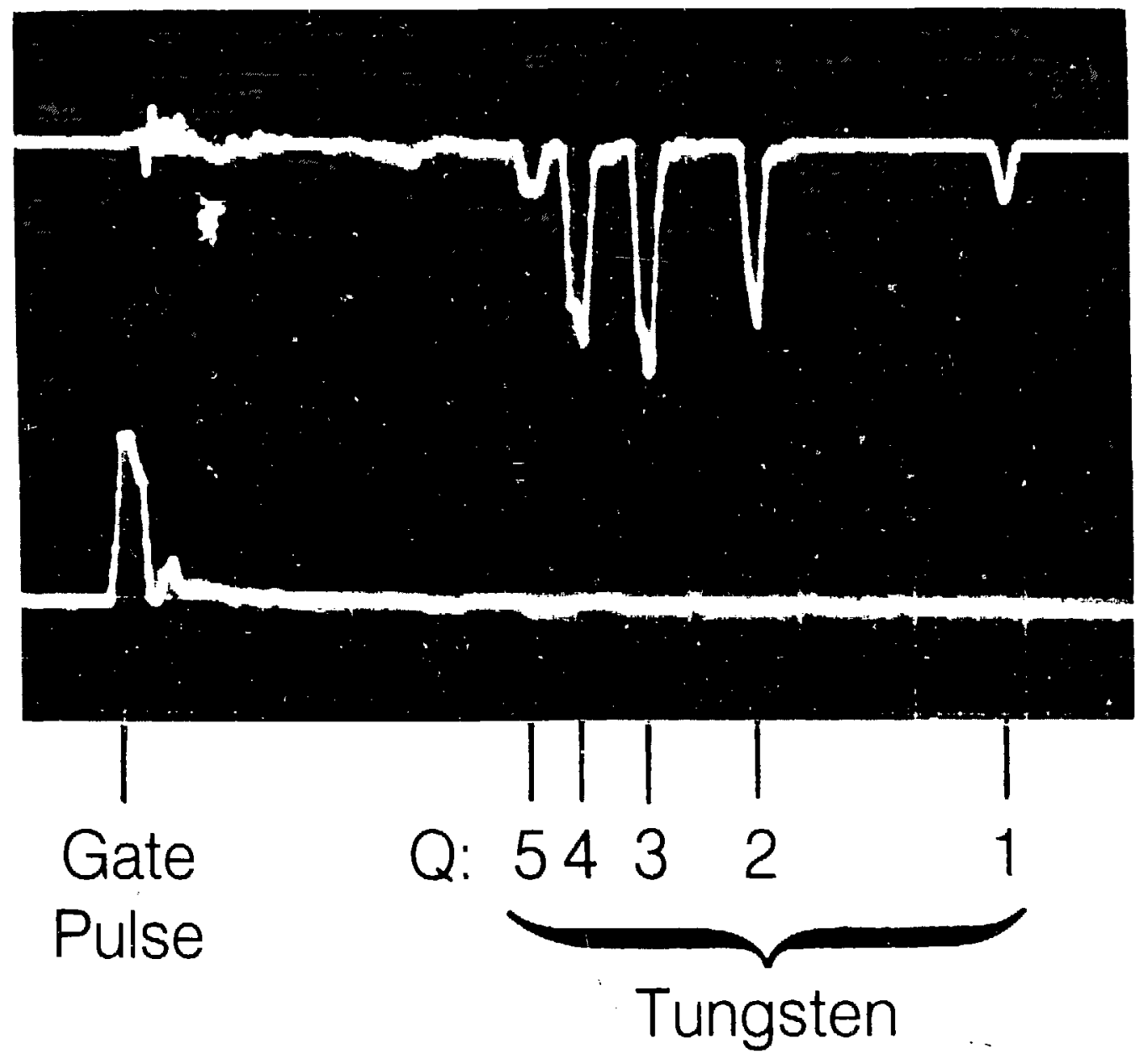

XBB 865-3963

Fig. 19 TOF spectrum for tungsten 


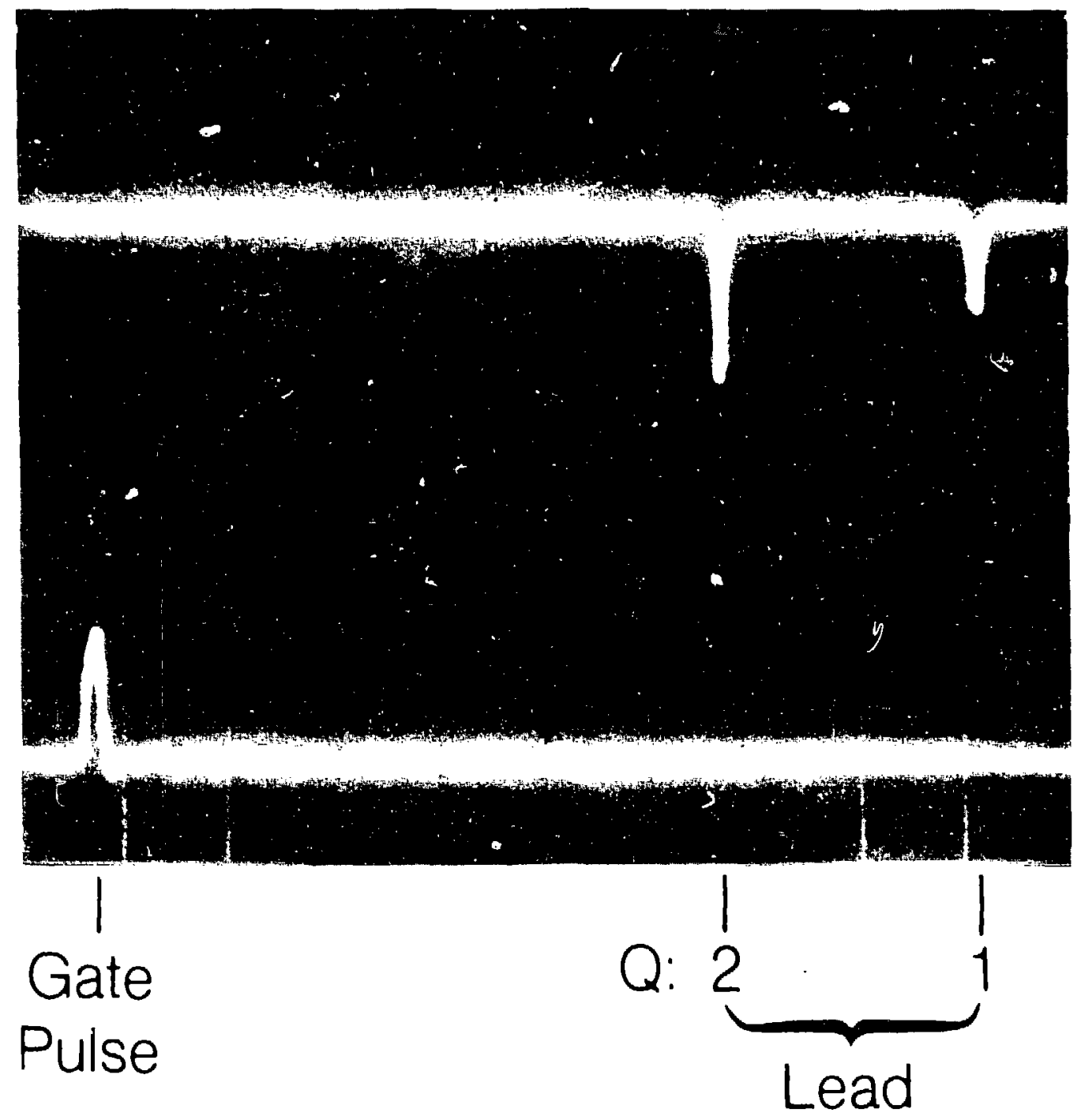

XBB $861-295$

Fig. 20 TOF spectrum for lead 


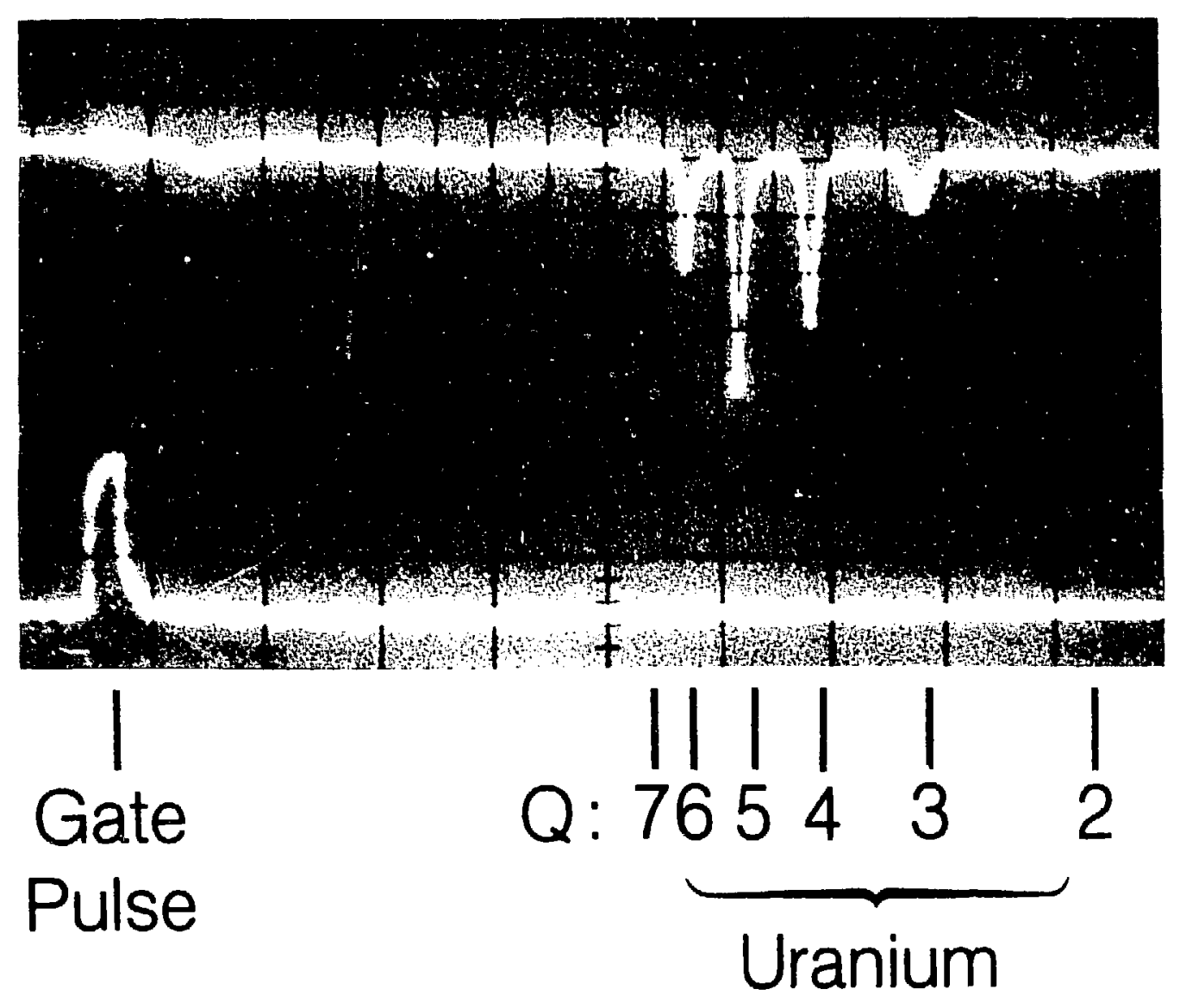

XBB $854-3522$

Fig. 21 TOF spectrum for uranium 


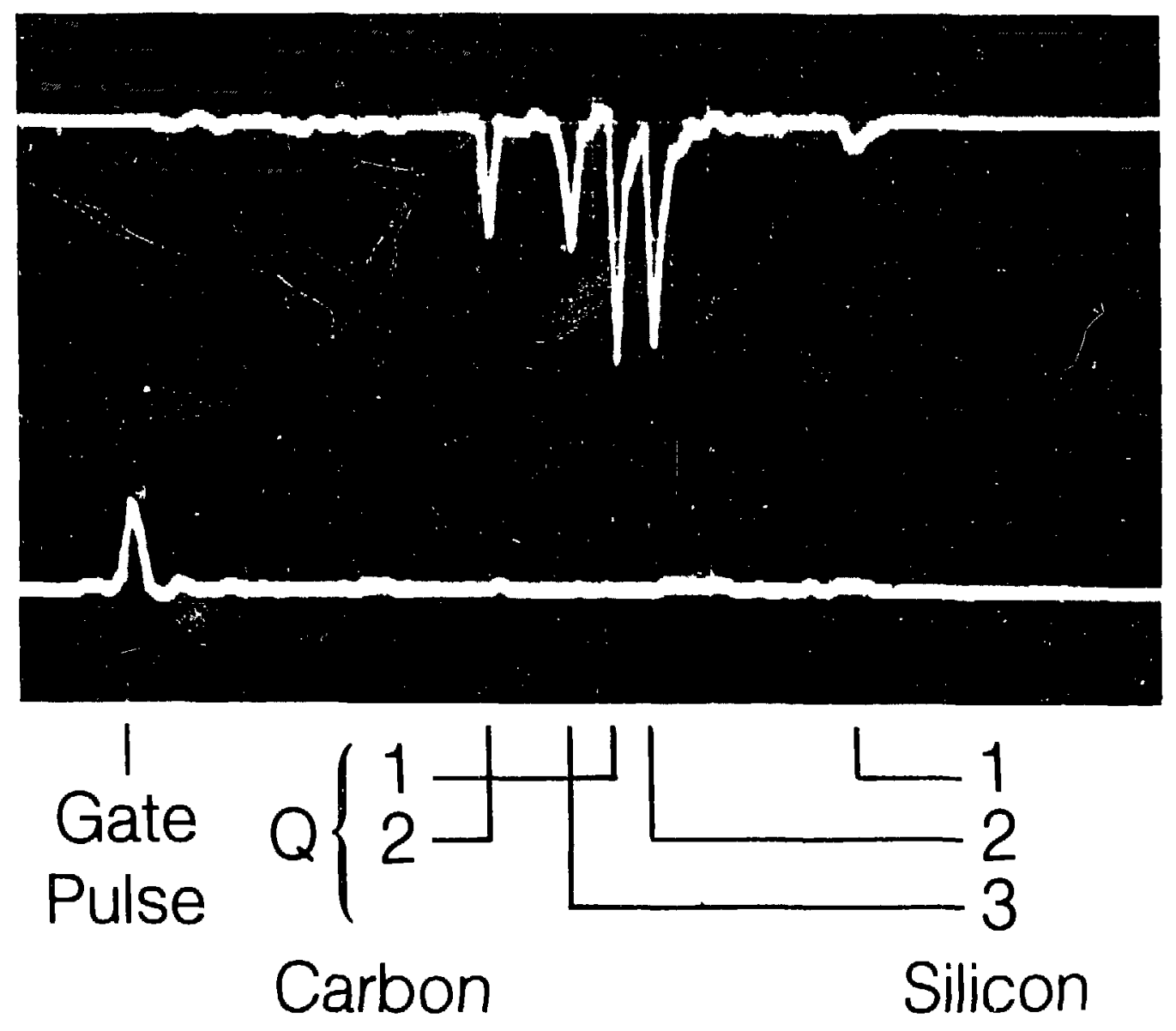

XBB $\quad 865-4260$

Fig. 22 TOF spectrum for silicon carbide 


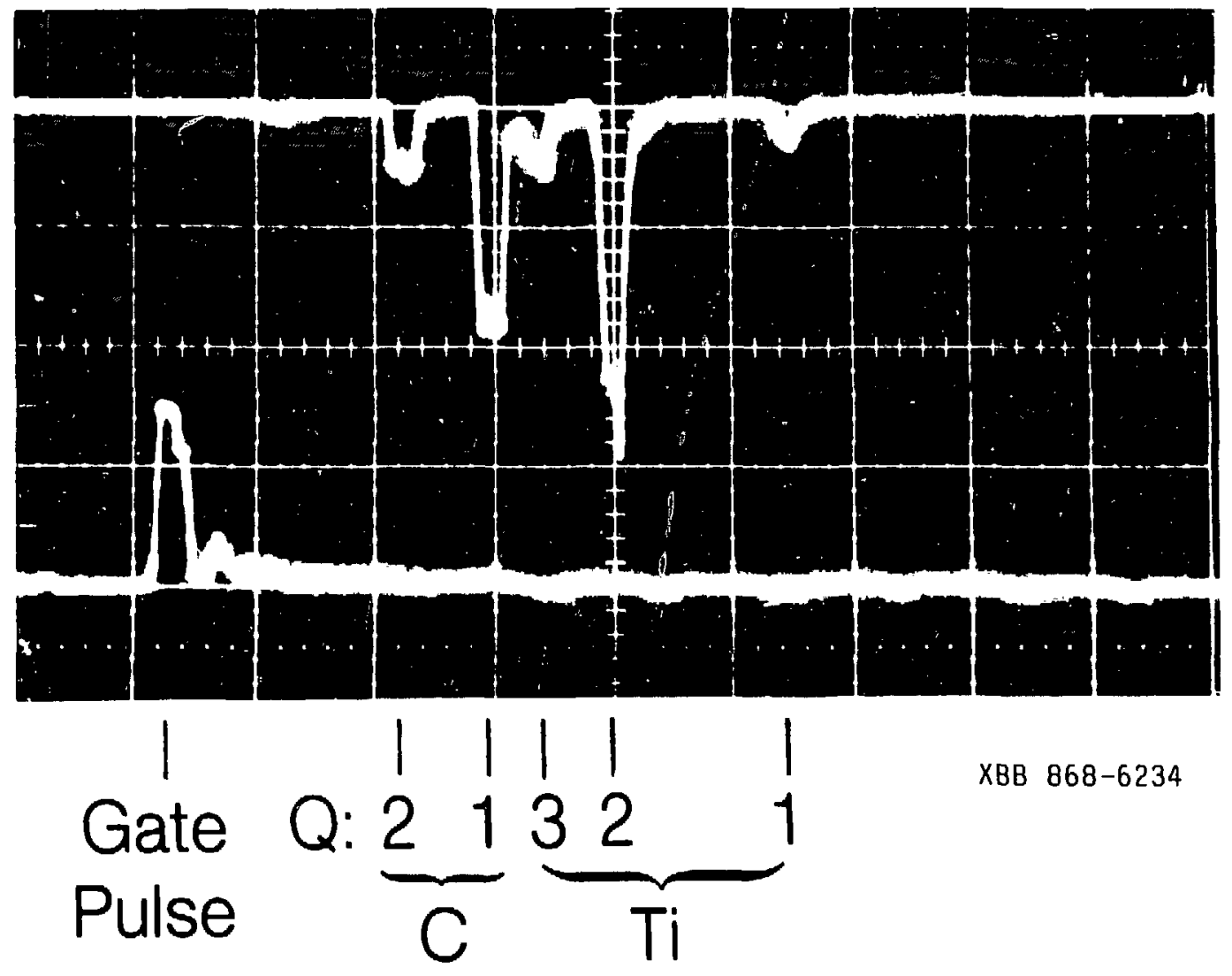

Fig. 23 TOF spectrum for titanium carbide 


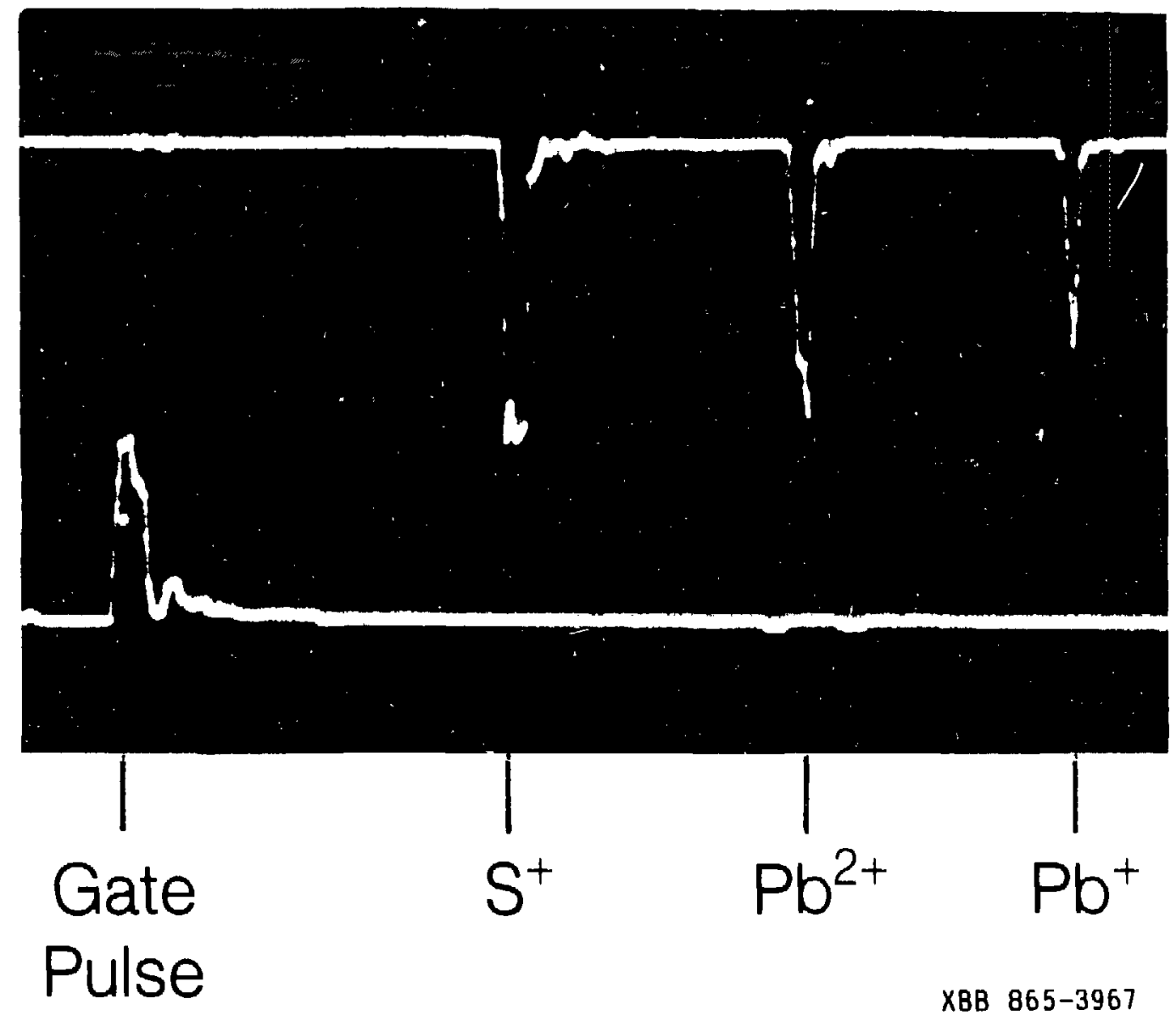

Fig. 24 TOF spectrum for lead sulfide 


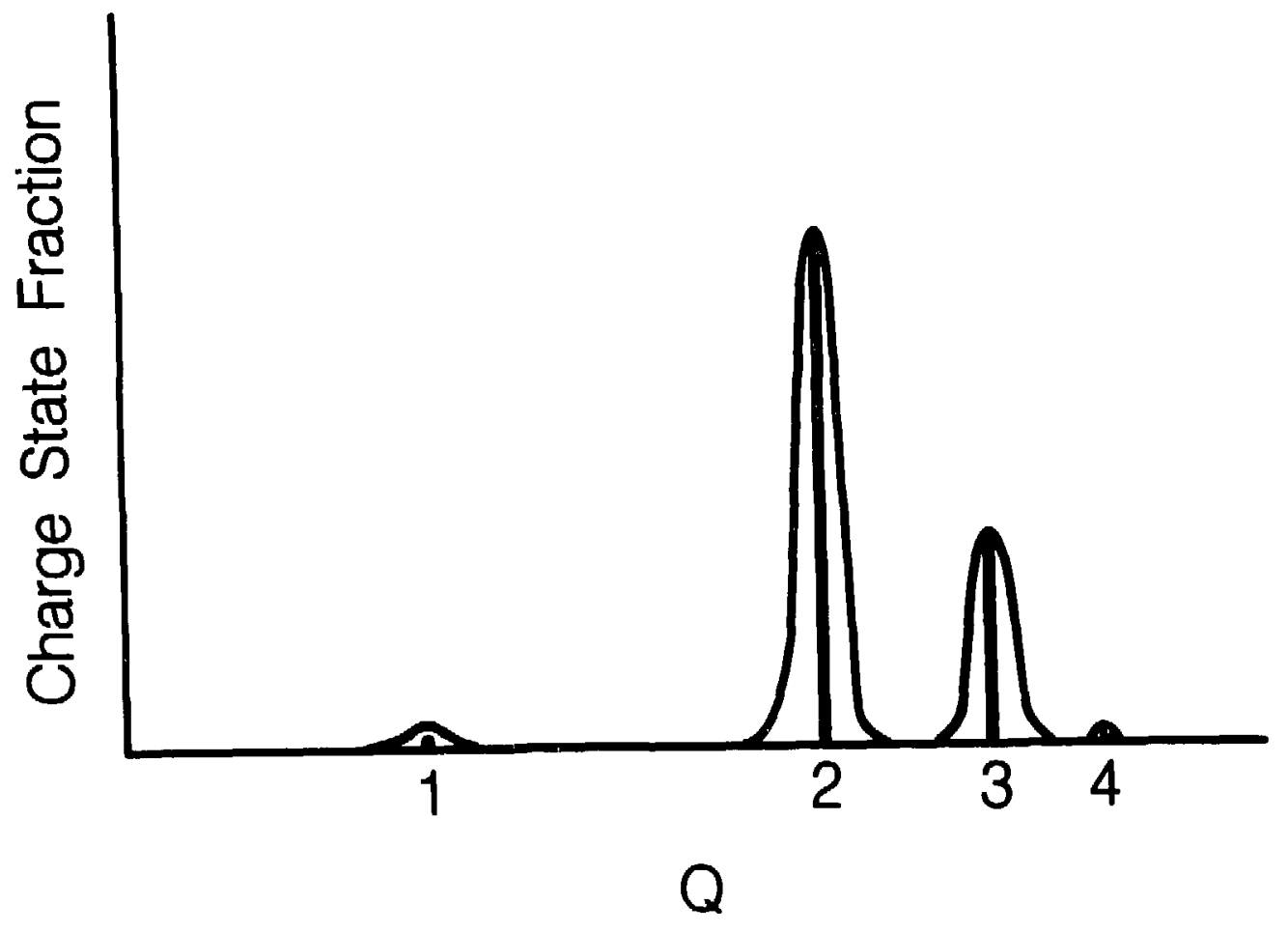

XBL 8610-9653

Fig. 25 Measured and calculated charge state distributions for titanium. The experimental CSO has been taken from Figure 8; the theoretical CSD, indicated by the vertical lines, has been taken from Figure 3 at the time indicated. 

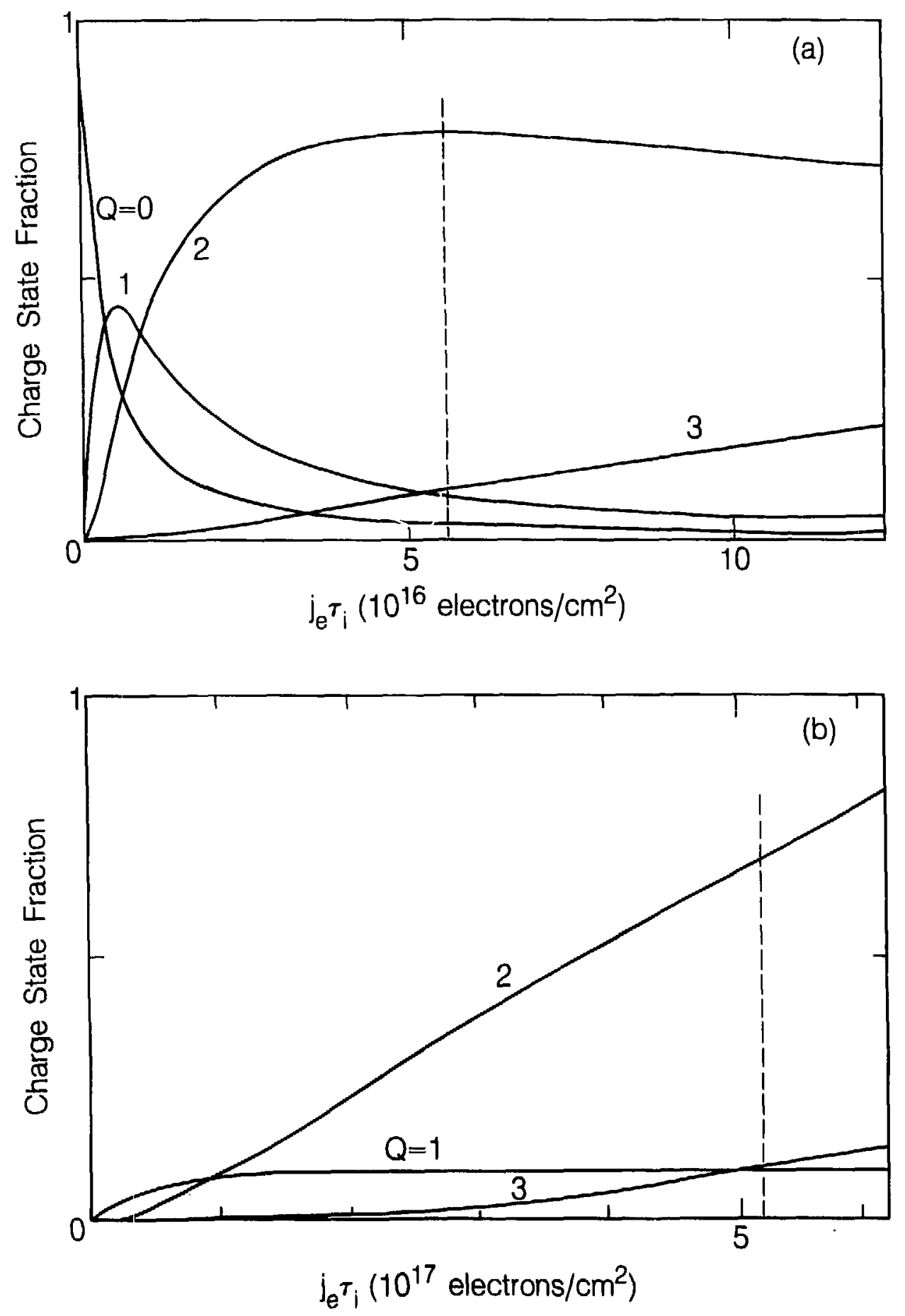

XBL B610-9657

Fig. 26 Charge state evolution predicted for gadolinium for two different sets of plasma parameters:

(a) monoenergetic with $E_{e}=20 \mathrm{eV}$, Gaussian density distribution, no fresh neutral injection:

(b) Maxwellian with $\mathrm{T}_{\mathrm{e}}=3.5 \mathrm{eV}$, uniform density distribution, steady injection of fresh neutrals.

In both cases a fit can be found to the measurements at the indicated values of $j_{e^{\tau} i}$. 


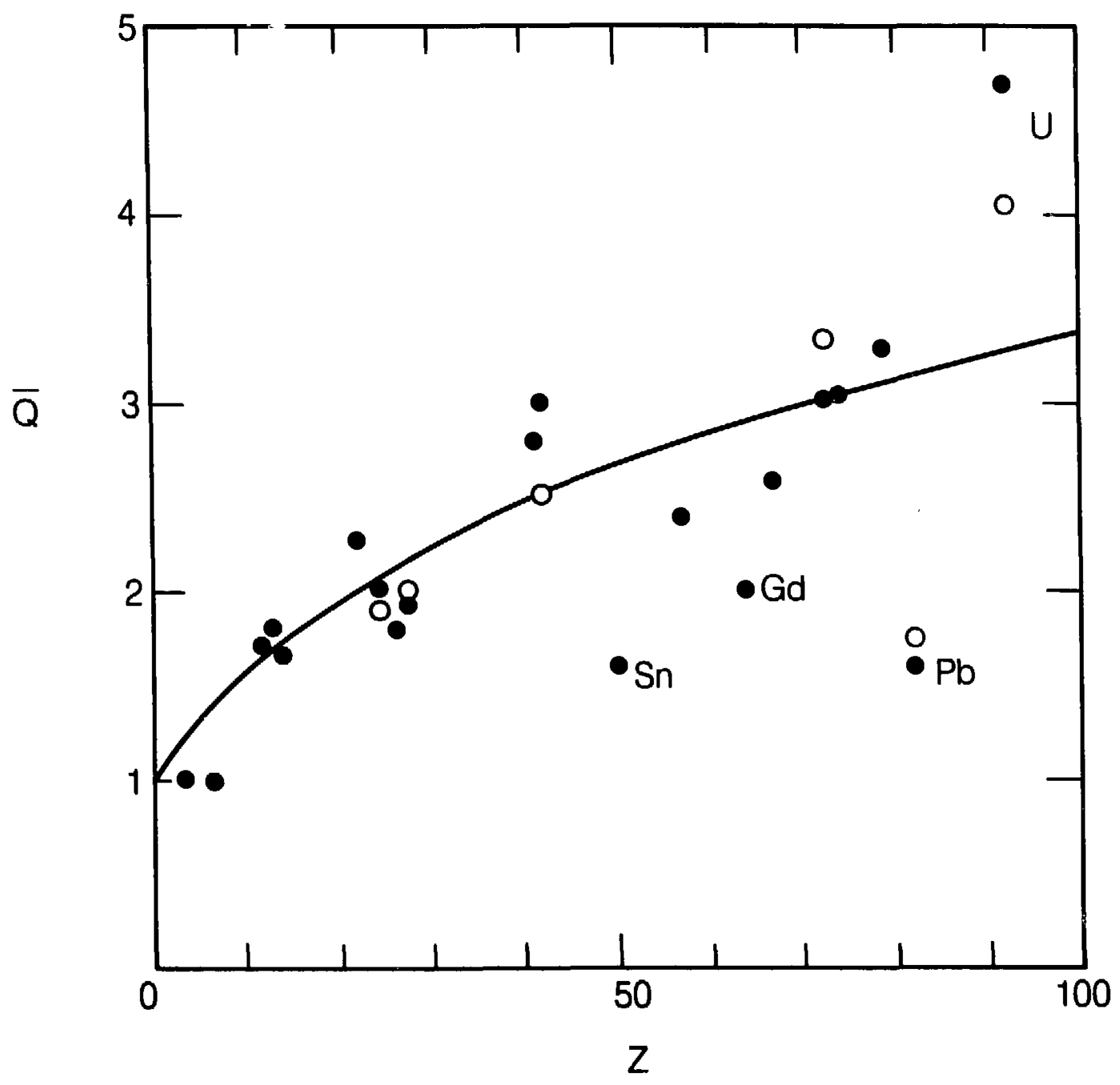

XBL 8610.9654

Fig. 27 Mean charge state, $\vec{Q}$, as a function of atomic number $z$. The experimental data are indicated by full circles. Predictions of the model based on the measured arc voltage and the Lotz cross sections are indicated by empty circles. The smooth curve is the function $\overline{0}=0.722^{1 / 3}$. 
This report was done with support from the Department of Energy. Any conclusions or opinions expressed in this report represent solely those of the author(s) and not necessarily those of The Regents of the University of California, the Lawrence Berkeley Laboratory or the Department of Energy.

Reference to a company or product name does not imply approval or recommendation of the product by the University of Califomia or the U.S. Department of Energy to the exclusion of oriers that may be suitable. 